\title{
Effelsberg H I observations of compact high-velocity clouds
}

\author{
T. Westmeier, C. Brüns, and J. Kerp
}

\author{
Radioastronomisches Institut der Universität Bonn, Auf dem Hügel 71, 53121 Bonn, Germany \\ e-mail: twestmei@astro.uni-bonn.de
}

Received 6 May 2004 / Accepted 24 November 2004

\begin{abstract}
We have mapped 11 compact high-velocity clouds (CHVCs) in the 21-cm line emission of neutral, atomic hydrogen, using the Effelsberg 100-m radio telescope. The aim of our observations was to study the overall distribution of the warm neutral medium of CHVCs with high sensitivity. The achieved baseline rms of $\sigma_{\mathrm{rms}} \approx 50 \mathrm{mK}$ at the original $2.6 \mathrm{~km} \mathrm{~s}^{-1}$ velocity resolution allows us to search for evidence of ram-pressure interaction with the ambient medium. In addition, we have obtained spectra along an appropriate axis across each CHVC with longer integration times and denser angular sampling. These deep slices with $\sigma_{\text {rms }} \approx 25 \ldots 35 \mathrm{mK}$ allow us to determine the column density profile in greater detail as well as the velocity and line width gradient across each cloud.

The most outstanding result of our observations is the complexity of the H I column density distribution and the line profiles of the investigated CHVCs. We have found only one cloud with a spherically-symmetric appearance. Among the remaining clouds we observe head-tail structures, bow-shock shapes, and objects with irregular shapes. These complex morphologies in combination with the obtained physical parameters suggest that ram-pressure interactions with an ambient medium may play a significant role in shaping some of the CHVCs from our sample. These results are consistent with a circumgalactic distribution of CHVCs with typical distances of the order of $100 \mathrm{kpc}$. The pressure of the ambient medium might also stabilise CHVCs in addition to their own gravitational potential.
\end{abstract}

Key words. ISM: clouds - Galaxy: halo - Galaxy: evolution - galaxies: Local Group - galaxies: ISM

\section{Introduction}

High-velocity clouds (HVCs) were first detected by Muller et al. (1963) in the 21-cm line emission of neutral, atomic hydrogen. HVCs are gas clouds which are characterised by high radial velocities incompatible with a participation in Galactic rotation. Over the past decades, there have been a large number of definitions concerning the velocities of HVCs. Following a suggested approach by Wakker (1991), their radial velocities have to be at least $50 \mathrm{~km} \mathrm{~s}^{-1}$ higher than the maximum radial velocities allowed for Galactic gas in the same direction according to a simple model of Galactic rotation. Apart from several small clouds, HVCs appear as large, homogeneous complexes, some of them spanning tens of degrees across the sky (see Wakker \& van Woerden 1997 for a detailed review).

Among the numerous hypotheses proposed for the origin and distribution of HVCs (Wakker \& van Woerden 1997), the Local Group hypothesis has become the most discussed during recent years. In 1999, Blitz et al. argued that the observed properties of HVCs were consistent with a distribution throughout the entire Local Group after excluding some of the large HVC complexes and the Magellanic Stream. Furthermore, they suggested that HVCs might be the so-called missing dark-matter satellites predicted by cosmological cold dark matter (CDM) models (Klypin et al. 1999; Moore et al. 1999). These models predict many more dark-matter haloes for the Local Group than can be directly observed in the form of dwarf galaxies. Blitz et al. (1999) argued that these dark-matter haloes might not have formed stars on a grand scale and could, therefore, be identified with a population of HVCs spread across the entire Local Group.

Following the approach of Blitz et al. (1999), Braun \& Burton (1999) defined a subclass of compact, isolated HVCs on the basis of the Leiden/Dwingeloo Survey of Galactic neutral hydrogen (LDS, Hartmann \& Burton 1997). They compiled a catalogue of 66 so-called compact highvelocity clouds (CHVCs), characterised by angular sizes of less than $2^{\circ} \mathrm{FWHM}$ and spatial isolation and separation from neighbouring H I emission. Braun \& Burton (1999) found the statistical properties of these CHVCs to be consistent with a distribution throughout the entire Local Group with typical distances of the order of $1 \mathrm{Mpc}$. CHVCs would then be quite large and massive objects with sizes of about $15 \mathrm{kpc}$ and typical H I masses of a few times $10^{7} M_{\odot}$.

An improved CHVC catalogue with 67 objects on the basis of the LDS was published by de Heij et al. (2002b), using an automated search routine. In addition, Putman et al. (2002) extracted 179 CHVCs from the Hi Parkes All-Sky Survey (HIPASS, Barnes et al. 2001). Both catalogues for the northern and southern sky were then combined by de Heij et al. (2002c) for an all-sky catalogue of 216 CHVCs (30 clouds were located in the overlap region between LDS and HIPASS). 
First doubts about a Local Group population of CHVCs arose with the non-detection of similar H I clouds in other galaxy groups. Zwaan (2000) investigated five galaxy groups with the Arecibo telescope to search for intragroup clouds. From his non-detection he concluded that the Local Group scenario proposed by Blitz et al. (1999) can be ruled out with $>99 \%$ confidence level if the Local Group is typical among the investigated groups. A similar survey was conducted by Pisano et al. (2004) who studied three galaxy groups with the Parkes 64-m telescope and the Australia Telescope Compact Array (ATCA). They were not successful in detecting any H I clouds similar to CHVCs, concluding that the known population of CHVCs has to be located within $160 \mathrm{kpc}$ from the Milky Way.

Further support for a circumgalactic population of CHVCs comes from Brüns et al. (2001) who discovered a pronounced head-tail structure of CHVC $125+41$, suggesting that gas had been stripped off the cloud by ram-pressure interaction with the surrounding medium. This suggests a location of the cloud in the vicinity of the Milky Way. By applying the virial theorem to a compact clump within the cloud, Brüns et al. (2001) constrained the distance of CHVC $125+41$ to $d \approx 130 \mathrm{kpc}$ (in the case of no additional molecular gas) which is consistent with a circumgalactic population of CHVCs. Head-tail structures are also observed towards most of the large HVC complexes. Brüns et al. (2000) systematically searched the LDS for distinct HVCs with $N_{\mathrm{HI}} \geq 10^{19} \mathrm{~cm}^{-2}$. They found that about $20 \%$ of the 252 clouds in their catalogue show a head-tail structure suggesting the presence of ram-pressure interaction between these HVCs and the ambient medium.

A circumgalactic CHVC population is also supported by hydrodynamical simulations of Sternberg et al. (2002) who considered CHVCs as spherically-symmetric, pressuresupported gas clouds confined by a gravitationally dominant dark-matter halo. They simulated both a Local Group population of CHVCs with a typical distance of $750 \mathrm{kpc}$ and a circumgalactic distribution with a distance of $150 \mathrm{kpc}$. Sternberg et al. (2002) compared their simulations with the observed properties of CHVCs according to high-resolution observations with the Westerbork Synthesis Radio Telescope (WSRT) by Braun \& Burton (2000) and Arecibo observations by Burton et al. (2001). They found the observed properties to be consistent with the results of their circumgalactic model so that CHVCs might be associated with an extended Galactic corona. At a typical distance of $150 \mathrm{kpc}$ the average H I mass of CHVCs would be $M_{\mathrm{HI}} \approx 3 \times 10^{5} M_{\odot}$. Furthermore, a diffuse Galactic corona gas can provide the external pressure required to additionally support CHVCs and to account for head-tail structures like the one observed in the case of CHVC 125+41.

The results of Sternberg et al. (2002) also outline observational strategies to distinguish between a Local Group origin of CHVCs and a circumgalactic CHVC population. In the circumgalactic case, their simulations predict a sharp drop in the H I column density below $N_{\mathrm{HI}} \approx 5 \times 10^{18} \mathrm{~cm}^{-2}$ due to ionisation by the strong intergalactic radiation field in the vicinity of the Galaxy. In addition, the circumgalactic model predicts possible gas wings with H I column densities below $10^{18} \mathrm{~cm}^{-2}$. In the Local Group scenario the HI colum density shows a smooth decline at the edge of the clouds and no extended wings are expected. Sensitive observations with the $9^{\prime}$ HPBW of the 100-m telescope in Effelsberg should, thus, allow us to evaluate the distribution of CHVCs by investigating the column density profile across a sample of objects in detail. Another aim of our observations is the search for head-tail structures among CHVCs which would suggest the presence of an ambient medium and could provide an additional constraint on the distance and distribution of CHVCs.

This paper is organised as follows: in Sect. 2 we describe how the 11 investigated CHVCs were chosen. Section 3 explains the entire data acquisition and reduction process. In Sect. 4 we present the results of our survey and summarise the physical parameters of our 11 CHVCs. In Sect. 5 we discuss the implications of our results for the origin and the properties of CHVCs. Sect. 6 summarises our results and conclusions.

\section{Sample selection}

10 of the 11 CHVCs were selected from the Braun \& Burton (1999) catalogue on the basis of previous, less sensitive Effelsberg H I observations of 41 CHVCs (Westmeier 2003) by two criteria. First, all clouds still had to be classified as CHVCs in the improved catalogue by de Heij et al. (2002b). Second, we selected only those clouds with an H I peak column density ratio of $N_{\mathrm{HI}}^{\mathrm{Eff}} / N_{\mathrm{HI}}^{\mathrm{LDS}} \geq 3$ between the Effelsberg data and the LDS data. A high column density ratio implies that the clouds contain compact substructure which is unresolved with the 25-m Dwingeloo telescope (36' HPBW). The obtained column density ratios are all well below the value expected for point sources so that our 11 CHVCs must all be partly resolved with the 100-m Effelsberg telescope ( $9^{\prime}$ HPBW). Thus, the latter criterion selects the most compact clouds for re-investigation which are not completely resolved with the existing LDS data and worth being re-observed with the higher angular resolution of the 100-m telescope.

The remaining object, CHVC 218+29, was selected from the de Heij et al. (2002b) catalogue to obtain a complete coverage of the available sidereal time interval. CHVC $218+29$ was attractive because of its northern latitude, its small angular size and its unusually narrow $\mathrm{HI}$ lines of only $7 \mathrm{~km} \mathrm{~s}^{-1} F W H M$ according to the de Heij et al. (2002b) catalogue.

\section{Data acquisition and reduction}

A map of $11 \times 11$ spectra on a $9^{\prime}$ grid (beam-by-beam sampling) was observed for most clouds, resulting in a field of view of $1.5^{\circ} \times 1.5^{\circ}$. The central position was derived from previous, less sensitive Effelsberg observations (Westmeier 2003) so that the object should be centred in the map. Each H I spectrum was integrated for $3 \mathrm{~min}$ in the in-band frequency switching mode, using the 1024-channel autocorrelator. The bandwidth was $6.3 \mathrm{MHz}$, resulting in a velocity resolution of $2.6 \mathrm{~km} \mathrm{~s}^{-1}$. Signal and reference spectra were shifted by $\pm 1.4 \mathrm{MHz}$ relative to the central frequency. The advantage of this in-band frequency switching method is that the $\mathrm{HI}$ line of the CHVC is included in both the signal and reference spectra so that the entire integration time is used for detection of the astronomical signal, resulting in a typical baseline rms of $\sigma_{\text {rms }} \approx 50 \mathrm{mK}$ 
at the original velocity resolution of $2.6 \mathrm{~km} \mathrm{~s}^{-1}$. The maps allow us to determine the overall morphology of the CHVCs and to identify possible head-tail structures or other signs of rampressure distortion.

Furthermore, we intended to compare the column density profiles of CHVCs with the predictions of the numerical simulations by Sternberg et al. (2002). For this purpose, we observed additional positions along the apparent symmetry axis of each CHVC with a longer integration time of typically 10 min per spectrum and a denser angular sampling of $4.5^{\prime}$ or $6.4^{\prime}$ between two adjacent spectra. These deep slices allow us to investigate the H I column density profile in more detail and with higher sensitivity. The typical baseline rms along the deep slices is $\sigma_{\mathrm{rms}} \approx 25 \ldots 35 \mathrm{mK}$ at the original $2.6 \mathrm{~km} \mathrm{~s}^{-1}$ velocity resolution. As the deep slices in most cases had to be observed prior to a detailed analysis of the maps, there are two cases in which the slices were unfortunately not perfectly placed along the symmetry axis of the corresponding clouds.

In the case of CHVC $218+29$ we used a slightly different procedure. The object turned out to be very compact and elongated so that we chose a map size of $15 \times 9$ spectra on a $4.5^{\prime}$ grid, corresponding to a field of view of roughly $70^{\prime} \times 40^{\prime}$. Furthermore, some H I lines of CHVC 218+29 turned out to be so narrow $\left(\approx 4 \mathrm{~km} \mathrm{~s}^{-1} F W H M\right)$ that the velocity resolution of $2.6 \mathrm{~km} \mathrm{~s}^{-1}$ could not sufficiently resolve them. Therefore, we had to observe CHVC $218+29$ with a smaller bandwidth of $3.2 \mathrm{MHz}$, leading to a velocity resolution of $1.3 \mathrm{~km} \mathrm{~s}^{-1}$. This made the use of the in-band frequency switching method inappropriate so that we had to observe CHVC $218+29$ in the normal frequency switching mode, resulting in a slightly higher baseline rms of $\sigma_{\mathrm{rms}}=88 \mathrm{mK}$ at the original $1.3 \mathrm{~km} \mathrm{~s}^{-1}$ velocity resolution.

All spectra were calibrated using the S7 standard calibration source (Kalberla et al. 1982). The statistical uncertainties in the derived calibration factors are in the range of $1 \ldots 3 \%$ for each observing session. Next, the standard stray radiation correction was applied to the spectra (Kalberla et al. 1980). Because of their high radial velocities most CHVCs are only marginally affected by Galactic stray radiation. However, a few of our CHVCs have radial velocities of $\left|v_{\text {LSR }}\right| \lesssim 200 \mathrm{~km} \mathrm{~s}^{-1}$ which is in the regime of Galactic HI gas towards some directions so that a stray radiation correction was in general recommended and performed for all spectra. After calibration and stray radiation correction all spectra were available in the form of brightness temperature $T_{\mathrm{B}}$ versus radial velocity in the local standard-of-rest frame $v_{\mathrm{LSR}}$.

The baseline correction for each spectrum was done by visual inspection, using the GILDAS package. As a result of the relatively narrow spectral lines of the CHVCs, the line windows could be set visually and a polynomial - usually of order 3 - was then subtracted from the spectra. To obtain the physical parameters of our CHVCs (e.g. H I column density, line width and radial velocity of the gas) a single Gaussian was fit to each spectrum. Finally, only those H I lines with a brightness temperature of $T_{\mathrm{B}} \geq 3 \sigma_{\mathrm{rms}}$ were selected for further analysis and all other spectra were rejected. Unless otherwise noted, all maps and slices presented in this paper are based on the results of these Gaussian fits. Only the column density maps were derived from the zeroth moment of the spectra under the general assumption that the optical depth of the gas is negligible. In some cases, the HI lines disclose a two-component structure indicating the presence of a cold and a warm gas phase. If so, we additionally fitted two Gaussian components to the spectra in order to study the properties of the two gas phases separately. Some examples of two-component line profiles are shown in Fig. 1.

\section{Results}

In this section we describe the physical parameters of the observed CHVCs. Section 4.1 gives a general overview of the properties of our CHVC sample. Sections 4.2 to 4.5 address the properties of the individual clouds in more detail.

\subsection{Overall properties of the $11 \mathrm{CHVCs}$}

One motivation for our observations was the search for headtail structures like the ones found in the case of CHVC 125+41 (Brüns et al. 2001) and in many HVC complexes (Brüns et al. 2000). It turned out that only one object, CHVC 148-82, has a spherically-symmetric appearance. All other CHVCs exhibit a more or less complex and irregular morphology. We identified 6 CHVCs with either a head-tail structure or a bow-shock shape which in many cases is suggestive of ram-pressure interaction between the CHVCs and the ambient medium. The remaining clouds appear irregular in the sense that they do not exhibit a simple radial or axial symmetry. In Sects. 4.2 to 4.5 we describe the properties of the individual CHVCs divided into the four morphological categories mentioned above. This morphological classification, however, is subjective and not based on a quantitative analysis. Nonetheless, we applied it to structure the description of the individual CHVCs with the objective of improving the readability of the paper. Our attempt to quantify the different morphologies on the basis of ellipse fits was not successful because of the relatively poor sampling of the CHVCs even with the $100-\mathrm{m}$ telescope. A single pixel could significantly influence the fits and, thus, the morphological classification of a cloud.

The derived physical parameters of the 11 investigated CHVCs are summarised in Table 1. With the exception of CHVC 218+29 the radial velocities of the clouds are all negative in both the local standard-of-rest frame (LSR) and the Galactic standard-of-rest frame (GSR). This is simply based on a selection effect: The motion of Galactic rotation is superposed on the radial velocities of the CHVCs so that CHVCs in the northern sky have predominantly negative radial velocities in the LSR frame while CHVCs in the southern hemisphere have mainly positive radial velocities (also see Braun \& Burton 1999). We can account for the contribution of Galactic rotation by converting the radial velocities into the GSR frame. The asymmetry between positive and negative velocities, however, remains so that even in the GSR frame CHVCs in the northern hemisphere must have a net negative radial velocity. This means that the net radial velocity of CHVCs is of no use if we do not consider a homogeneous all-sky survey. Even in the latter case gas clouds of different origin (e.g. fragments of 

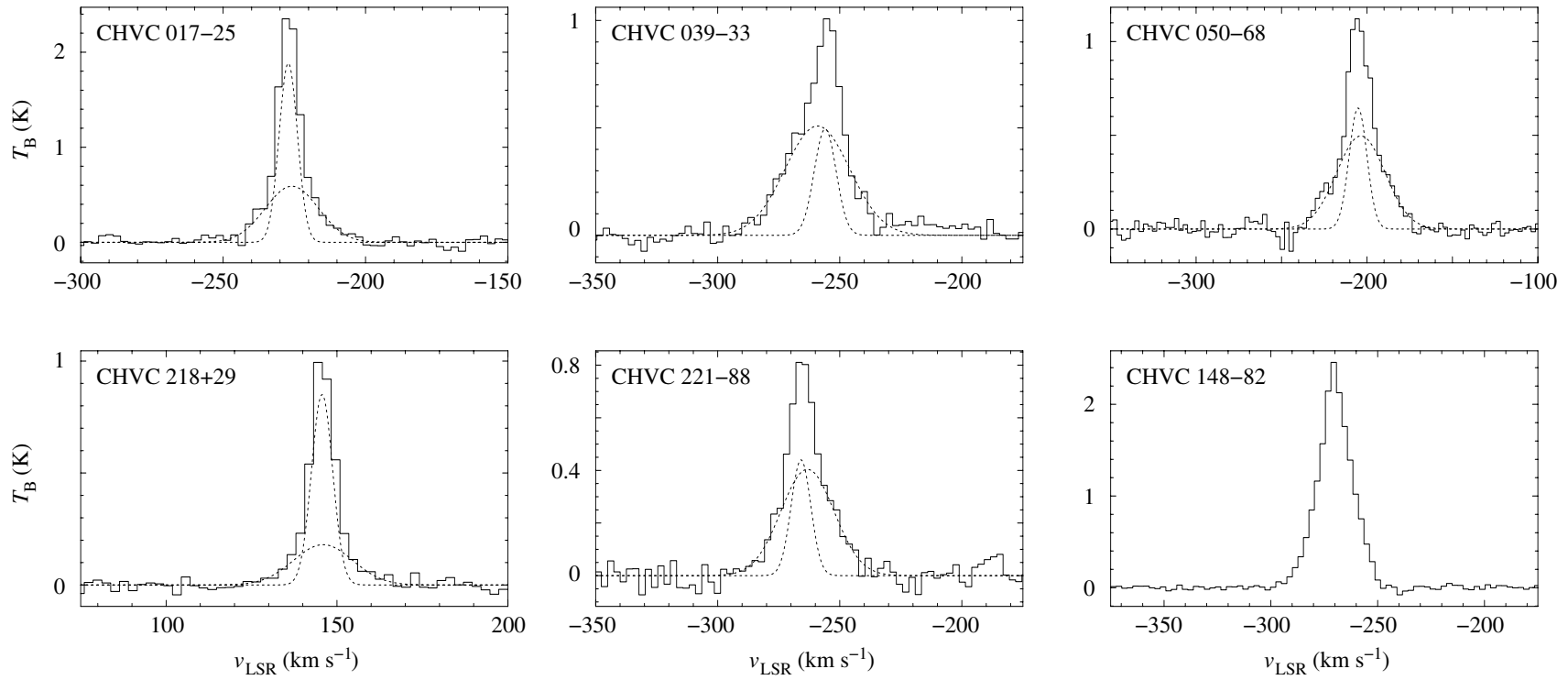

Fig. 1. Example spectra of the five CHVCs with two-component line profiles and of CHVC 148-82. The dotted curves represent a decomposition of the spectra into two Gaussian components. The superposition of a broad and a narrow Gaussian component indicates the presence of two more or less distinct phases of warm and cold neutral gas. In the case of CHVC 148-82 a separation into two Gaussian components is not useful, although the narrow cusp on top of the $\mathrm{HI}$ lines towards the centre of the cloud again indicates the existence of a compact core of cold neutral gas.

Table 1. Physical parameters of the 11 observed CHVCs. $l$ and $b$ are the Galactic longitude and latitude of the column density maximum, $\alpha$ and $\delta$ the corresponding equatorial coordinates, $v_{\mathrm{LSR}}$ and $v_{\mathrm{GSR}}$ the column density weighted average radial velocities in LSR and GSR frames, $\Delta v$ the average line width $(F W H M), T_{\mathrm{B}}$ the observed peak brightness temperature, $N_{\mathrm{H}}$ the H I peak column density, and $e$ the ellipticity of the cloud. The last two rows give the mean value $\langle x\rangle$ and the standard deviation $\sigma_{x}$ for each parameter $x$.

\begin{tabular}{lccrrrrrr}
\hline \hline $\begin{array}{l}\text { Name } \\
(\text { CHVC } l \pm b)\end{array}$ & $\alpha(2000)$ & $\delta(2000)$ & $\begin{array}{r}v_{\mathrm{LSR}} \\
\left(\mathrm{km} \mathrm{s}^{-1}\right)\end{array}$ & $\begin{array}{r}v_{\mathrm{GSR}} \\
\left(\mathrm{km} \mathrm{s}^{-1}\right)\end{array}$ & $\begin{array}{r}\Delta v \\
\left(\mathrm{~km} \mathrm{~s}^{-1}\right)\end{array}$ & $\begin{array}{r}T_{\mathrm{B}} \\
(\mathrm{K})\end{array}$ & $\begin{array}{r}N_{\mathrm{HI}} \\
\left(10^{19} \mathrm{~cm}^{-2}\right)\end{array}$ & $e$ \\
\hline CHVC 016.8-25.2 & $19^{\mathrm{h}} 59^{\mathrm{m}} 05^{\mathrm{s}}$ & $-24^{\circ} 42^{\prime}$ & -228 & -171 & 14 & 3.1 & 5.6 & 0.38 \\
CHVC 032.1-30.7 & $20^{\mathrm{h}} 41^{\mathrm{m}} 40^{\mathrm{s}}$ & $-14^{\circ} 07^{\prime}$ & -308 & -207 & 30 & 1.3 & 6.0 & 0.33 \\
CHVC 039.0-33.2 & $21^{\mathrm{h}} 00^{\mathrm{m}} 58^{\mathrm{s}}$ & $-09^{\circ} 51^{\prime}$ & -262 & -147 & 22 & 1.6 & 8.0 & 0.42 \\
CHVC 039.9+00.6 & $19^{\mathrm{h}} 02^{\mathrm{m}} 03^{\mathrm{s}}$ & $+06^{\circ} 28^{\prime}$ & -278 & -137 & 32 & 0.7 & 4.5 & 0.49 \\
CHVC 050.4-68.4 & $23^{\mathrm{h}} 24^{\mathrm{m}} 14^{\mathrm{s}}$ & $-19^{\circ} 00^{\prime}$ & -195 & -133 & 27 & 1.3 & 4.7 & 0.52 \\
CHVC 147.5-82.3 & $01^{\mathrm{h}} 05^{\mathrm{m}} 02^{\mathrm{s}}$ & $-20^{\circ} 05^{\prime}$ & -269 & -254 & 22 & 2.2 & 8.0 & 0.32 \\
CHVC 157.1+02.9 & $04^{\mathrm{h}} 49^{\mathrm{m}} 01^{\mathrm{s}}$ & $+49^{\circ} 20^{\prime}$ & -184 & -98 & 22 & 0.9 & 3.6 & 0.58 \\
CHVC 172.1-59.6 & $02^{\mathrm{h}} 21^{\mathrm{m}} 57^{\mathrm{s}}$ & $-05^{\circ} 40^{\prime}$ & -235 & -219 & 28 & 0.9 & 4.2 & 0.68 \\
CHVC 218.1+29.0 & $08^{\mathrm{h}} 44^{\mathrm{m}} 18^{\mathrm{s}}$ & $+08^{\circ} 41^{\prime}$ & +145 & +27 & 6 & 2.8 & 3.2 & 0.58 \\
CHVC 220.5-88.2 & $00^{\mathrm{h}} 59^{\mathrm{m}} 28^{\mathrm{s}}$ & $-27^{\circ} 21^{\prime}$ & -258 & -263 & 22 & 1.0 & 3.7 & 0.58 \\
CHVC 357.8+12.4 & $16^{\mathrm{h}} 54^{\mathrm{m}} 25^{\mathrm{s}}$ & $-23^{\circ} 43^{\prime}$ & -159 & -167 & 27 & 1.5 & 6.4 & 0.60 \\
\hline$\langle x\rangle$ & & & -203 & -161 & 23 & 1.6 & 5.3 & 0.50 \\
$\sigma_{x}$ & & & \pm 124 & \pm 81 & \pm 7 & \pm 0.8 & \pm 1.7 & \pm 0.12 \\
\hline
\end{tabular}

the Magellanic Stream or gas clouds of Galactic origin) might significantly influence the obtained mean radial velocity of the CHVC population so that the implications of the mean radial velocity of CHVCs must be considered with great care.

There is a wide range of different velocity gradients across the 11 investigated CHVCs. The two extreme cases are CHVC $218+29$ which shows velocity variations of only $4 \mathrm{~km} \mathrm{~s}^{-1}$ and CHVC $358+12$ with a total gradient of about $85 \mathrm{~km} \mathrm{~s}^{-1}$. In a few cases, rapid velocity changes seem to be induced by the presence of multiple gas components along the line of sight with different radial velocities. This is likely the case for CHVC 050-68, where we observe a velocity gradient of $60 \mathrm{~km} \mathrm{~s}^{-1}$ across an angular distance of only $20^{\prime}$. Broad and complex line profiles in this area suggest that the gradient is induced by individual gas components along the line of sight. In other cases, velocity variations are fairly regular with Gaussian line profiles, so that the velocity gradients can be interpreted as a sign of rotation of the clouds. This is in particular the case for CHVC $358+12$ where the velocity profile along the major axis of the cloud resembles the rotation curve of a galaxy.

The average line width of the investigated CHVCs is $23 \pm$ $7 \mathrm{~km} \mathrm{~s}^{-1} F W H M$. The narrowest lines by far are found in the 
case of CHVC 218+29, measuring around $4 \mathrm{~km} \mathrm{~s}^{-1}$ FWHM at the eastern edge of the cloud. This corresponds to an upper limit in kinetic gas temperature of about $350 \mathrm{~K} .{ }^{1}$ The largest line widths of up to $60 \mathrm{~km} \mathrm{~s}^{-1} F W H M$ are found in CHVC $358+12$, but the complex line profiles in this case suggest that such large line widths are caused by the superposition of multiple gas components with different radial velocities. In some CHVCs, line profiles show a twocomponent structure. A narrow line component seems to be superposed on a broad component, indicating the presence of two phases of cold neutral medium (CNM) and warm neutral medium (WNM). Such two-component line profiles can be seen in CHVC 017-25, CHVC 039-33, CHVC 050-68, CHVC 218+29 and CHVC 221-88. Example spectra of these clouds are shown in Fig 1. In the case of CHVC 148-82 (lowerright panel), no clear two-component structure is present in the spectra. The shape of the spectral lines, however, is not perfectly Gaussian in the central parts of the cloud. A narrow cusp on top of the $\mathrm{HI}$ line indicates the presence of a compact cold core which is not resolved with the $9^{\prime}$ HPBW of the 100-m telescope. Evidence for a cold core in CHVC 148-82 was also found by de Heij et al. (2002a). Their high-resolution synthesis observations with the WSRT show a very compact clump with $\mathrm{HI}$ line widths of $\lesssim 10 \mathrm{~km} \mathrm{~s}^{-1} F W H M$.

The average HI peak column density of the CHVCs in the original catalogue by Braun \& Burton (1999) is $N_{\mathrm{HI}}=$ $2.1 \pm 1.6 \times 10^{19} \mathrm{~cm}^{-2}$. The observed peak column densities of our $11 \mathrm{CHVCs}$ are in the range of $N_{\mathrm{HI}} \approx 3 \ldots 8 \times 10^{19} \mathrm{~cm}^{-2}$ with a slightly larger average value of $5.3 \pm 1.7 \times 10^{19} \mathrm{~cm}^{-2}$. This is consistent with the value of $5 \times 10^{19} \mathrm{~cm}^{-2}$ following from the circumgalactic model of Sternberg et al. (2002). The peak brightness temperatures of the CHVCs in our survey range from $0.7 \mathrm{~K}$ to $3.1 \mathrm{~K}$ with an average of $1.6 \pm 0.8 \mathrm{~K}$.

The ellipticities of the clouds were determined by a second moment analysis following the procedure described by Banks et al. (1995). First, a clip level of $5 \times 10^{18} \mathrm{~cm}^{-2}$ was applied to the column density map of each cloud, corresponding to about the 3-sigma detection limit of our data. Next, the central position of the cloud was derived from the column density weighted first moment. Then we calculated the column density weighted second moments for each map from which we obtained the major and minor axis, $a$ and $b$, of the ellipse as described by Banks et al. (1995). From the major and minor axis we finally calculated the ellipticity $e$, using the definition $e=1-\frac{b}{a}$.

\subsection{Spherically-symmetric CHVCs}

\subsubsection{CHVC 148-82}

CHVC $148-82$ is the only cloud among our 11 investigated CHVCs which has an almost spherically-symmetric appearance. It is located close to the Galactic south pole and the Magellanic Stream. The radial velocity of CHVC 148-82, however, is different by about $170 \mathrm{~km} \mathrm{~s}^{-1}$ from the one

${ }^{1} T=m_{\mathrm{H}} \Delta v^{2} /(8 k \ln 2)$ according to the Maxwellian velocity distribution of an ideal gas where $m_{\mathrm{H}}$ is the mass of a hydrogen atom, $k$ the Boltzmann constant, and $\Delta v$ the $F W H M$ of the H I line. observed for the Magellanic Stream $\left(v_{\mathrm{LSR}} \approx-100 \mathrm{~km} \mathrm{~s}^{-1}\right.$, Brüns et al. 2005) in this area so that an association between both objects is unlikely. Figure 2 shows the distribution of H I column densities, line widths and radial velocities. We find a clear velocity gradient across the cloud, measuring roughly $8 \mathrm{~km} \mathrm{~s}^{-1}$ across an angular distance of about 45'. The distribution of line widths is consistent with a spherical symmetry of CHVC 148-82 as the HI lines in the centre of the cloud are typically narrower than those at the edge. This is what we would expect for a spherically-symmetric gas cloud with decreasing gas temperature towards the centre.

The results of the deep and more detailed observations performed along the slice indicated by the crosses in Fig. 2a are presented in Fig. 3. The column density profile confirms the symmetric appearance of CHVC 148-82 although towards the northern edge of the cloud the column density decreases more rapidly than towards the southern edge. This slight asymmetry is already indicated in the map in Fig. 2a. Unfortunately, the slice was not exactly chosen along the velocity gradient of CHVC 148-82. Nonetheless, the somewhat irregular velocity gradient is well traced by the data. The line width profile across the deep slice clearly shows the systematically smaller line widths in direction of the centre of CHVC 148-82 which suggests the existence of cold gas in the centre of the cloud. Although a distinct cold gas component cannot directly be seen in the spectra, its existence is indicated by narrow cusps on top of the HI lines in the direction of the cloud's centre (see Fig. 1). These cusps are a direct hint of the existence of a compact core of cold neutral gas which is not resolved with the $9^{\prime}$ HPBW of the Effelsberg telescope. This compact core was directly detected by de Heij et al. (2002a) with high-resolution WSRT observations.

\subsection{Head-tail CHVCs}

Four CHVCs from our sample exhibit a head-tail structure, suggesting the presence of ram-pressure interaction between these CHVCs and the ambient medium. Maps of the integrated column density and the distribution of radial velocities and line widths are shown in Fig. 4. The results of the deep and more detailed observations performed along the slice indicated by the crosses in Fig. 4a are presented in Fig. 5 as a function of relative position along the slice.

\subsubsection{CHVC 017-25}

CHVC 017-25 is located in the vicinity of the two HVC complexes GCP and GCN (Galactic Centre Positive/Negative). At first glance, the head-tail structure of CHVC 017-25 does not seem very pronounced. The column density map shows that the spacings between contour lines are noticeably wider towards the north-western edge of the cloud, indicating the existence of a diffuse, faint gas tail in this direction. In the H I column density distribution along the deep slice the head-tail structure of CHVC 017-25 can clearly be seen. At the south-eastern edge there is a sharp increase in column density while towards the north-western edge we see a faint and extended tail. 

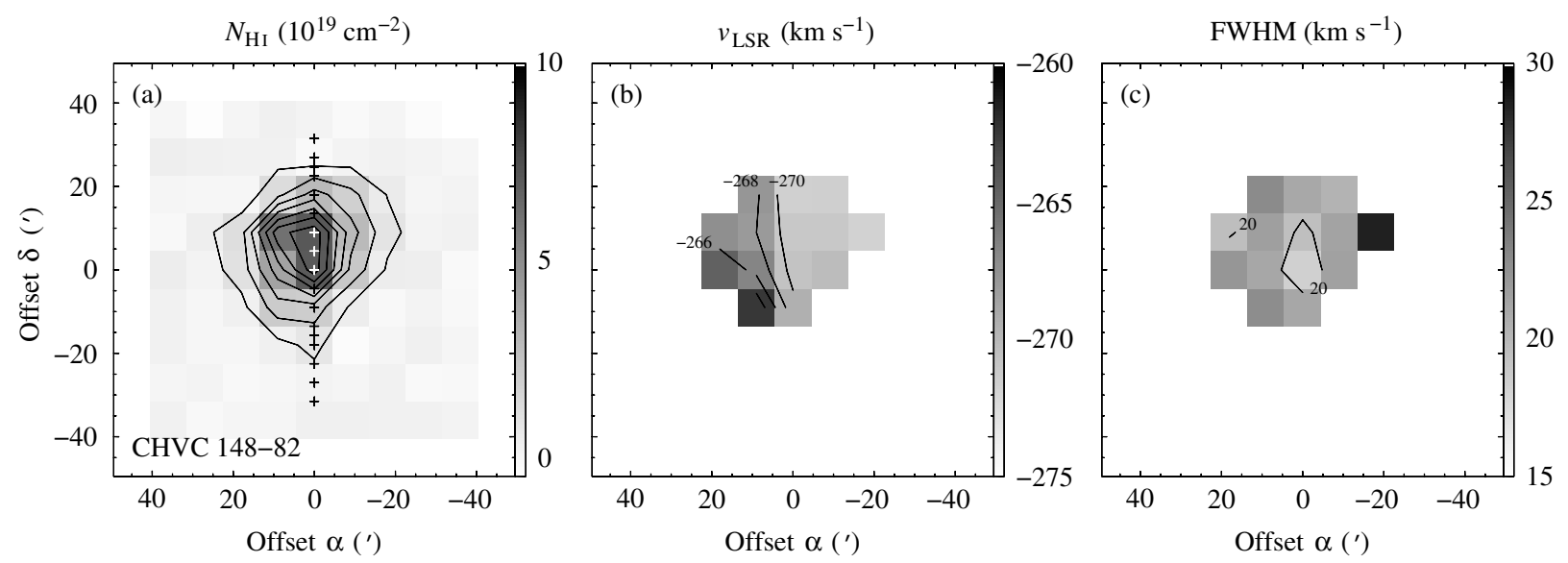

Fig. 2. CHVC 148-82 as described in Sect. 4.2. a) Integrated H I column density map. Contours start at $5 \times 10^{18} \mathrm{~cm}^{-2}$ with an increment of $1 \times 10^{19} \mathrm{~cm}^{-2}$. The crosses indicate the positions of individual spectra along the deep slice (see Fig. 3). b) Distribution of LSR radial velocities of the gas. c) Distribution of line widths $(F W H M)$.

The behaviour of radial velocities is not completely regular; however, there is a general north-south gradient of about $5 \mathrm{~km} \mathrm{~s}^{-1}$. Along the deep slice, radial velocities show a semi-periodic variation without any clear trend. The observed $\mathrm{H}$ I line widths show a clear gradient with quite narrow lines in the south-eastern part of the cloud. The narrowest lines at the south-eastern edge measure only about $7 \mathrm{~km} \mathrm{~s}^{-1} F W H M$ corresponding to an upper limit for the gas temperature of about $1100 \mathrm{~K}$. In the direction of the extended tail of CHVC 017-25 the line widths increase noticeably, reaching values around $20 \mathrm{~km} \mathrm{~s}^{-1} F W H M$ which corresponds to an upper limit for the gas temperature of roughly $9000 \mathrm{~K}$. These results suggest the separation of a cold and a warm gas component and substantiate the impression of a head-tail structure of CHVC 017-25. The obvious distortion of CHVC $017-25$ is discussed in more detail in Sect. 5.2.

\subsection{2. $\mathrm{CHVC} 032-31$}

CHVC 032-31 can be found in the direction of the HVC complex GCN which combines several smaller clouds with similar negative radial velocities. The head-tail structure of CHVC 032-31 is somewhat more pronounced than in the case of CHVC 017-25. At the north-western edge there is a sharp increase in column density while in the opposite direction the $\mathrm{HI}$ emission is much more extended and ends in a faint tail. Another remarkable feature is the isolated emission in the lower left corner of the map. With a maximum H I column density of $N_{\mathrm{H} \text { I }} \approx 10^{19} \mathrm{~cm}^{-2}$, this small cloud lies clearly above our detection limit. Within the significance of our data, it is not connected to the main body of CHVC 032-31, resulting in an upper limit $\left(3 \sigma_{\text {rms }}\right)$ for the column density of a potential bridge of roughly $2.5 \times 10^{18} \mathrm{~cm}^{-2}$ if a line width of $40 \mathrm{~km} \mathrm{~s}^{-1} F W H M$ is assumed. The radial velocity of the small isolated cloud is significantly lower (w.r.t. the absolute value) than the velocities observed across the main cloud, indicating a separation in both position and velocity. The H I lines across CHVC 032-31 are comparatively broad with a mean value of
$\langle\Delta v\rangle=30 \pm 7 \mathrm{~km} \mathrm{~s}^{-1}$. As in the case of CHVC 017-25, there is a strong line width gradient between head and tail.

\subsubsection{CHVC $040+01$}

CHVC $040+01$ is located very close to the Galactic plane. From the morphological point of view the head-tail structure of CHVC $040+01$ is the most pronounced among our $11 \mathrm{CHVCs}$. The column density map shows a compact head and an extended tail in the north-eastern direction. This structure can also be seen in the column density profile along the deep slice although the slice unfortunately is not perfectly aligned with the symmetry axis of CHVC $040+01$. Moreover, there is evidence of very faint and diffuse emission all across the map with intensities typically below $3 \sigma_{\text {rms }}$. This faint emission is particularly prominent in the western part of the map where column densities of up to $N_{\mathrm{HI}} \approx 1 \times 10^{19} \mathrm{~cm}^{-2}$ can be found. The observed radial velocities across CHVC 040+01 do not show a regular behaviour. There is no clear gradient, and radial velocities along the deep slice again show a semi-periodic variation as in the case of CHVC 017-25. The observed H I line widths are quite large, reaching about $40 \mathrm{~km} \mathrm{~s}^{-1}$ FWHM in the southwestern part of the cloud. Unlike in all other head-tail CHVCs, the broadest lines can be found in the vicinity of the head of CHVC 040+01. Along the tail, line widths decrease to around $30 \mathrm{~km} \mathrm{~s}^{-1}$ FWHM.

\subsubsection{CHVC $218+29$}

CHVC $218+29$ is one of the most remarkable objects among our 11 CHVCs because it reveals some extreme properties. It is highly elongated with a major axis of about $1^{\circ}$ but a minor axis of only $20^{\prime}$ with respect to the $5 \times 10^{18} \mathrm{~cm}^{-2}$ column density level. The head-tail structure is not very prominent but the deep column density profile shows a steep slope on one side of the cloud and a more moderate slope on the other side, forming a short and diffuse tail. Radial velocities across the object are nearly constant with $v_{\mathrm{LSR}} \approx+145 \mathrm{~km} \mathrm{~s}^{-1}$. Only towards the western edge is there a slight increase to about $+147 \mathrm{~km} \mathrm{~s}^{-1}$. The detected line widths are the smallest 

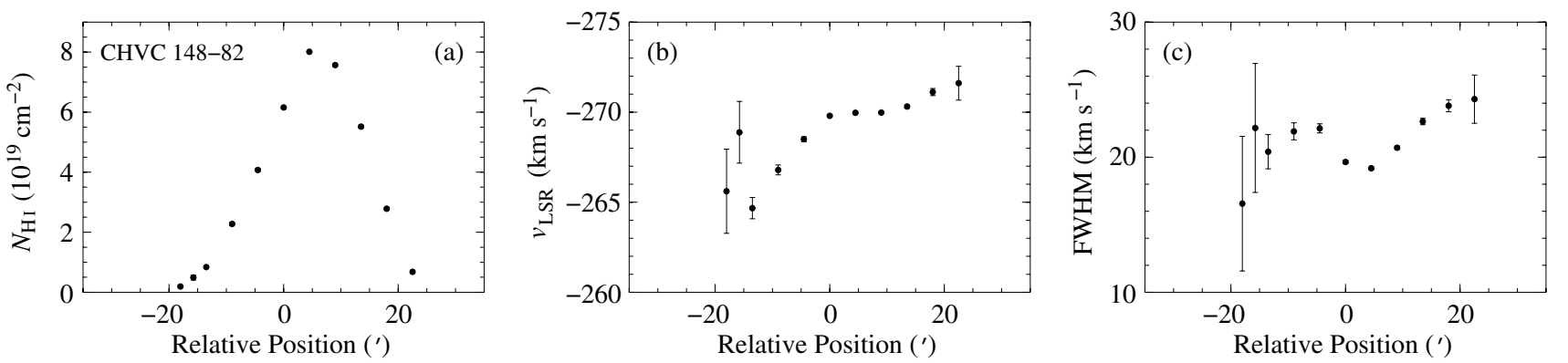

Fig. 3. CHVC 148-82 as described in Sect. 4.2. a) Integrated H I column density along the slice indicated by the crosses in Fig. 2a. b) Distribution of LSR radial velocities of the gas along the slice. c) Distribution of line widths $(F W H M)$ along the slice.

by far among the CHVCs in our sample. At the eastern edge we observe line widths of only around $4 \mathrm{~km} \mathrm{~s}^{-1} F W H M$, corresponding to an upper limit for the gas temperature of only about $350 \mathrm{~K}$. Moreover, we find a clear and systematic gradient along the major axis of CHVC $218+29$. Towards the eastern edge, line widths rise above $10 \mathrm{~km} \mathrm{~s}^{-1}$ FWHM. This prominent line width gradient again suggests the separation of a warm envelope and a cold gas component.

\subsection{Bow-shock shaped CHVCs}

Two CHVCs from our sample exhibit a bow-shock shape, suggesting the presence of ram-pressure interaction between these CHVCs and the ambient medium. Maps of the integrated column density and the distribution of radial velocities and line widths are shown in Fig. 6. The results of the deep and more detailed observations performed along the slice indicated by the crosses in Fig. 6a are presented in Fig. 7 as a function of relative position along the slice.

\subsubsection{CHVC $157+03$}

CHVC $157+03$ is located close to the Galactic plane and is characterised by a slightly condensed core and two symmetric gas wings north-east and south-west of the core. It is a nice example of a CHVC with a bow-shock shape. Both radial velocities and line widths follow the axial symmetry of the column density distribution. Across most of CHVC 157+03 the radial velocities of the gas are more or less the same with $v_{\text {LSR }} \approx-185 \mathrm{~km} \mathrm{~s}^{-1}$. Only towards the south-eastern edge do the radial velocities systematically decrease (w.r.t. their absolute values) by about $10 \mathrm{~km} \mathrm{~s}^{-1}$ which is clearly traced by the velocity gradient along the deep slice. A similar behaviour is observed for the H I line widths. Across most of the cloud the line widths are slightly above $20 \mathrm{~km} \mathrm{~s}^{-1} F W H M$, but towards the south-eastern edge they systematically rise above $30 \mathrm{~km} \mathrm{~s}^{-1}$ FWHM.

\subsubsection{CHVC 172-60}

The appearance of CHVC $172-60$ is similar to that of CHVC $157+03$ although the core is more condensed and the wings are clearly asymmetric. The wing in the eastern direction is significantly less extended than the wing in the western direction. As in the case of CHVC $157+03$, we observe a radial velocity gradient across the cloud which more or less follows the axial symmetry of the column density distribution. While we observe radial velocities around $-235 \mathrm{~km} \mathrm{~s}^{-1}$ or higher across most of CHVC 172-60, velocities systematically decrease below $-230 \mathrm{~km} \mathrm{~s}^{-1}$ towards the south-eastern and southwestern flanks of the cloud. The behaviour of the H I line widths, however, is different from that in CHVC 157+03. There is no clear increase in line width towards the southern edge of the cloud, but instead line widths grow noticeably towards the opposite side.

\subsection{Irregular CHVCs}

The remaining four $\mathrm{CHVCs}$ from our survey show an irregular structure in the sense that their morphologies and the distribution of radial velocities and line widths do not exhibit a simple spherical or axial symmetry. Maps of the integrated column density and the distribution of radial velocities and line widths are shown in Fig. 8. The results of the deep and more detailed observations performed along the slice indicated by the crosses in Fig. 8a are presented in Fig. 9 as a function of relative position along the slice.

\subsubsection{CHVC 039-33}

CHVC 039-33 can be found near the HVC complex GCN. At first glance, CHVC 039-33 appears to be a prime example of a head-tail CHVC. A very compact and condensed core is accompanied by an extended, diffuse gas tail in the southeastern direction. The distribution of radial velocities and line widths, however, does not follow this axial symmetry. At both the northern and southern edge the highest radial velocities of $v_{\text {LSR }} \approx-270 \mathrm{~km} \mathrm{~s}^{-1}$ are observed, while the lowest velocities of $v_{\text {LSR }} \gtrsim-260 \mathrm{~km} \mathrm{~s}^{-1}$ can be found along the eastern and western edge of CHVC 039-33. This "quadrupole" symmetry of radial velocities does not fit into the simple picture of a head-tail CHVC. Concerning the distribution of $\mathrm{H}$ I line widths, there is a strong gradient from south-west to north-east. In the south-western part of CHVC 039-33 we observe relatively narrow lines with $\Delta v \approx 15 \mathrm{~km} \mathrm{~s}^{-1} F W H M$. Towards the northeastern edge, the line widths reach $\Delta v \gtrsim 25 \mathrm{~km} \mathrm{~s}^{-1} F W H M$. Again, this clear gradient does not not follow the axial symmetry of the observed column density distribution but instead is perpendicular to the major axis of the cloud. The column density profile along the deep slice, which is shown in Fig. 9a, 

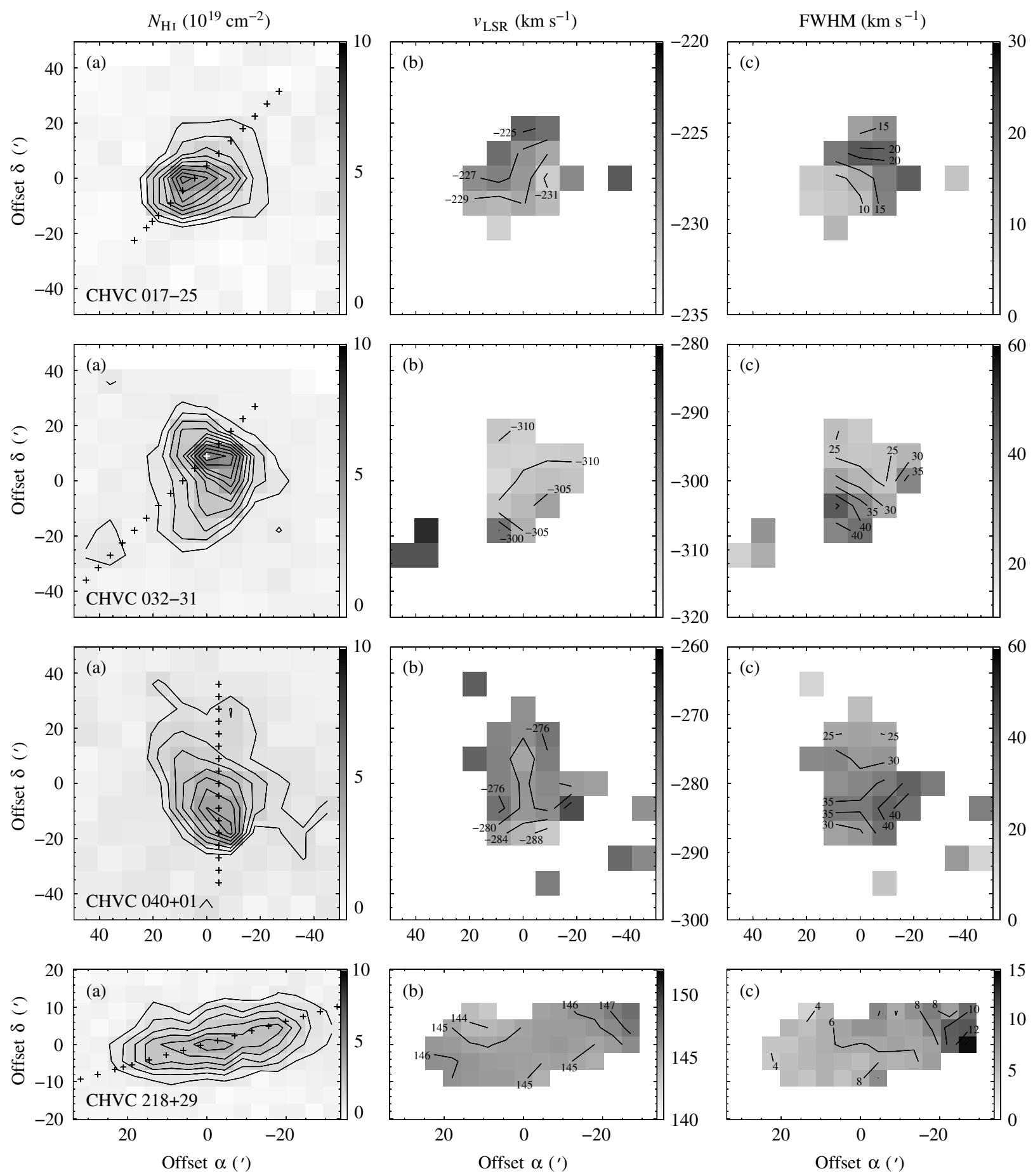

Fig. 4. Head-tail CHVCs as described in Sect. 4.3. a) Integrated H I column density map. Contours start at $5 \times 10^{18} \mathrm{~cm}^{-2}\left(1 \times 10^{19} \mathrm{~cm}^{-2}\right.$ in the case of CHVC 040+01) with an increment of $5 \times 10^{18} \mathrm{~cm}^{-2}$. The crosses indicate the positions of individual spectra along the deep slice (see Fig. 5). b) Distribution of LSR radial velocities of the gas. c) Distribution of line widths ( $F W H M$ ).

discloses a second maximum about $20^{\prime}$ south-east of the compact core. This smaller peak is not visible in the map because it is too compact and is located exactly in between the individual pointings of the map. In addition, the deep column density profile affirms the impression of a head-tail structure of CHVC 039-33. At the north-western edge, there is a sharp increase in column density, while towards the south-eastern edge we detect a very faint and extended tail. But the structure of CHVC 039-33 is more complicated than in the four cases of head-tail CHVCs discussed above. Therefore we classify it as an irregular CHVC.

\subsubsection{CHVC $050-68$}

CHVC 050-68 is located about $6^{\circ}$ away from the Magellanic Stream which has quite similar radial velocities in this area with $v_{\mathrm{LSR}} \approx-150 \ldots-200 \mathrm{~km} \mathrm{~s}^{-1}$ (Brüns et al. 2005). Because 

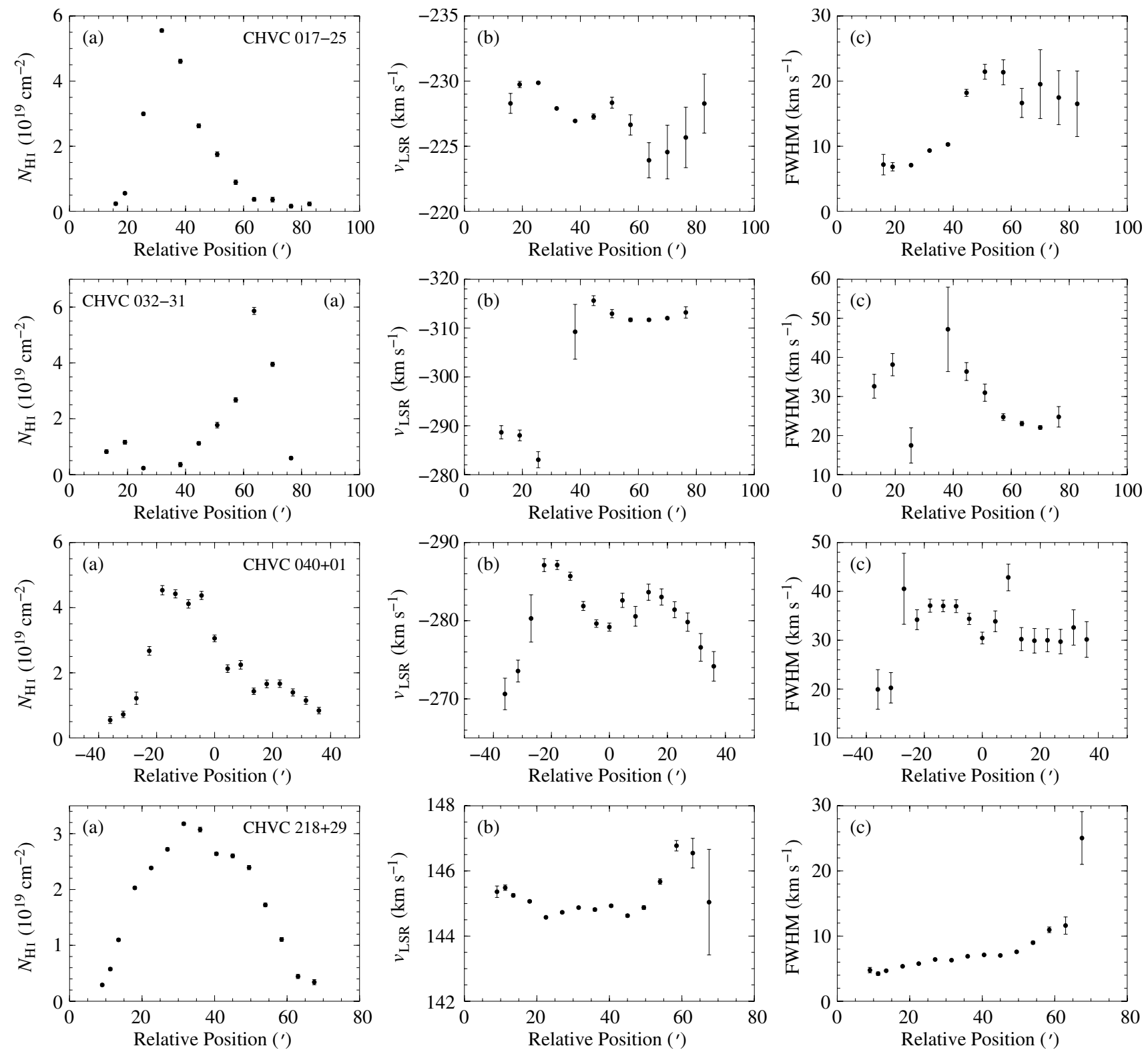

Fig. 5. Head-tail CHVCs as described in Sect. 4.3. a) Integrated H I column density along the slice indicated by the crosses in Fig. 4a. b) Distribution of LSR radial velocities of the gas along the slice. c) Distribution of line widths $(F W H M)$ along the slice.

of its extremely large radial velocity gradient CHVC 050-68 is among the most remarkable CHVCs in our sample. Another conspicuous feature is a narrow, elongated arm in the northeastern direction. This arm is rather faint, but at least one spectrum is clearly above our $3 \sigma_{\text {rms }}$ detection limit. The most amazing feature of CHVC 050-68, however, is its velocity gradient. At the eastern edge, we find a radial velocity of $v_{\text {LSR }} \approx-150 \mathrm{~km} \mathrm{~s}^{-1}$. Towards the centre of the cloud, the radial velocity increases to $v_{\mathrm{LSR}} \approx-210 \mathrm{~km} \mathrm{~s}^{-1}$ and then drops again to reach $v_{\mathrm{LSR}} \approx-180 \mathrm{~km} \mathrm{~s}^{-1}$ at the western edge. This means that in the eastern part of CHVC 050-68 we see a velocity gradient of $60 \mathrm{~km} \mathrm{~s}^{-1}$ across only about $20^{\prime}$. Across the western part of the cloud the velocity gradient still measures $30 \mathrm{~km} \mathrm{~s}^{-1}$ across $40^{\prime}$ and has the opposite sign. The somewhat smaller gradient in the western part of CHVC 050-68 might indicate a rotation of the cloud. This is reasonable because the line widths observed in this part are almost constant. Moreover, the observed line profiles are regular and reveal a clear two-component structure, indicating the presence of two distinct cold and warm gas phases (see Fig. 1). The eastern part of CHVC 050-68, however, is characterised by a sudden increase in line widths and complex, non-Gaussian line profiles. This indicates the presence of individual gas components with different radial velocities along the line of sight, whose superposition could give the impression of a strong velocity gradient across the eastern part of the cloud. This interpretation is supported by the extended, narrow arm in the north-eastern direction which exhibits radial velocities around $v_{\mathrm{LSR}} \approx-150 \mathrm{~km} \mathrm{~s}^{-1}$, consistent with the values detected at the eastern edge of CHVC 050-68. 

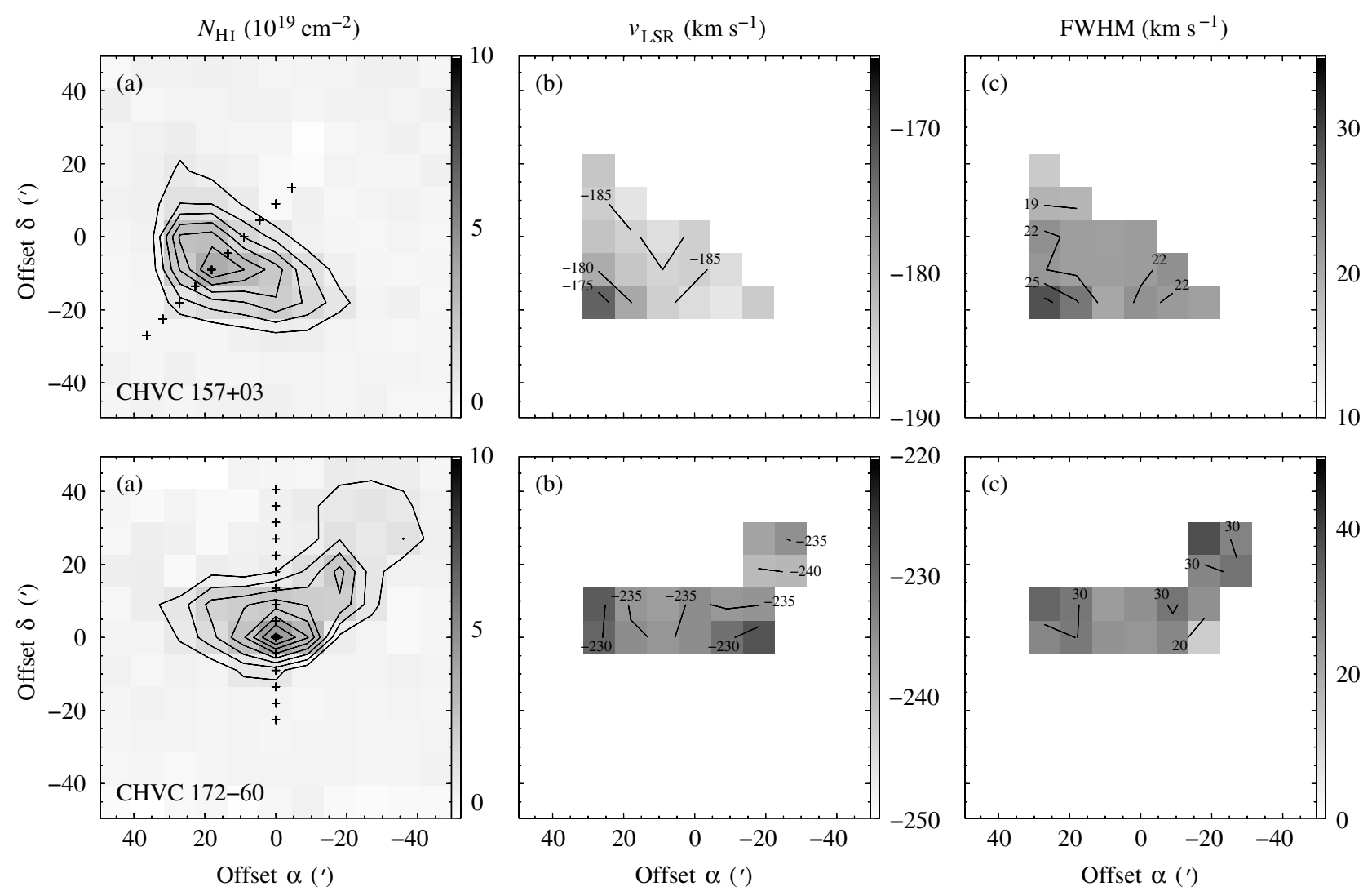

Fig. 6. Bow-shock shaped CHVCs as described in Sect. 4.4. a) Integrated H I column density map. Contours start at $5 \times 10^{18} \mathrm{~cm}^{-2}$ with an increment of $5 \times 10^{18} \mathrm{~cm}^{-2}$. The crosses indicate the positions of individual spectra along the deep slice (see Fig. 7). b) Distribution of LSR radial velocities of the gas. c) Distribution of line widths $(F W H M)$.

\subsubsection{CHVC 221-88}

CHVC 221-88 is located near the Galactic south pole and about $5^{\circ}$ away from the Magellanic Stream. Nonetheless, a connection between both objects is unlikely because the Magellanic Stream reveals completely different radial velocities in this area with $v_{\mathrm{LSR}} \approx-50 \mathrm{~km} \mathrm{~s}^{-1}$ (Brüns et al. 2005). CHVC 221-88 is among the most complex CHVCs investigated in our survey. It is quite elongated with a major axis of about $1^{\circ}$ and a minor axis between $20^{\prime}$ and $30^{\prime}$. The column density map and, in more detail, the deep column density profile reveal three clumps, the westernmost of which is the faintest. Additional emission is detected at the western edge of the map. This emission has radial velocities around $v_{\text {LSR }} \approx-210 \mathrm{~km} \mathrm{~s}^{-1}$ which is about $40 \ldots 50 \mathrm{~km} \mathrm{~s}^{-1}$ different from the velocities observed across the main part of CHVC 221-88. This means that despite their positional coincidence both clouds are kinematically clearly separated from each other. Unfortunately, we did not have enough time to extend our map in the western direction to investigate the second cloud in more detail. Focussing on the main cloud, its velocity structure is quite remarkable. While in the central and eastern parts of CHVC 221-88 radial velocities range between $v_{\text {LSR }} \approx-260 \ldots-265 \mathrm{~km} \mathrm{~s}^{-1}$, they suddenly drop to $v_{\mathrm{LSR}} \approx-250 \mathrm{~km} \mathrm{~s}^{-1}$ in the western part of the cloud. The velocity profile along the deep slice which is presented in Fig. 9b shows that this drop by about $15 \mathrm{~km} \mathrm{~s}^{-1}$ is extremely abrupt and occurs across only $9^{\prime}$ (which is the HPBW of the Effelsberg telescope's main beam). In the intermediate region between both radial velocity regimes the observed line widths suddenly increase, indicating an increased velocity dispersion along the line of sight. At first glance, the velocity profile is reminiscent of a rotation curve. But the shape of the spectral lines in the intermediate region clearly exhibits two distinct gas components so that the two parts of CHVC 221-88 with different radial velocities might be two separate clouds on the same line of sight rather than a single rotating cloud. The separate cloud at the western edge of the map supports this interpretation as it is also isolated in both velocity and position. It looks as if we see three individual clouds across the map with radial velocities decreasing (in absolute value) from east to west.

\subsubsection{CHVC $358+12$}

CHVC $358+12$ is located about $12^{\circ}$ north of the Galactic centre. It is the largest among our 11 investigated CHVCs and extends beyond the boundaries of our map in both the eastern and western direction. CHVC $358+12$ reveals a rather complex structure with a compact core in the centre and several fainter clumps and extensions all across the object. The most remarkable feature, however, is the radial velocity gradient. In the south-western part of CHVC $358+12$ radial velocities up to $v_{\text {LSR }} \approx-195 \mathrm{~km} \mathrm{~s}^{-1}$ are observed. Towards the north-eastern edge the radial velocities drop to $v_{\mathrm{LSR}} \approx-130 \mathrm{~km} \mathrm{~s}^{-1}$, and the 

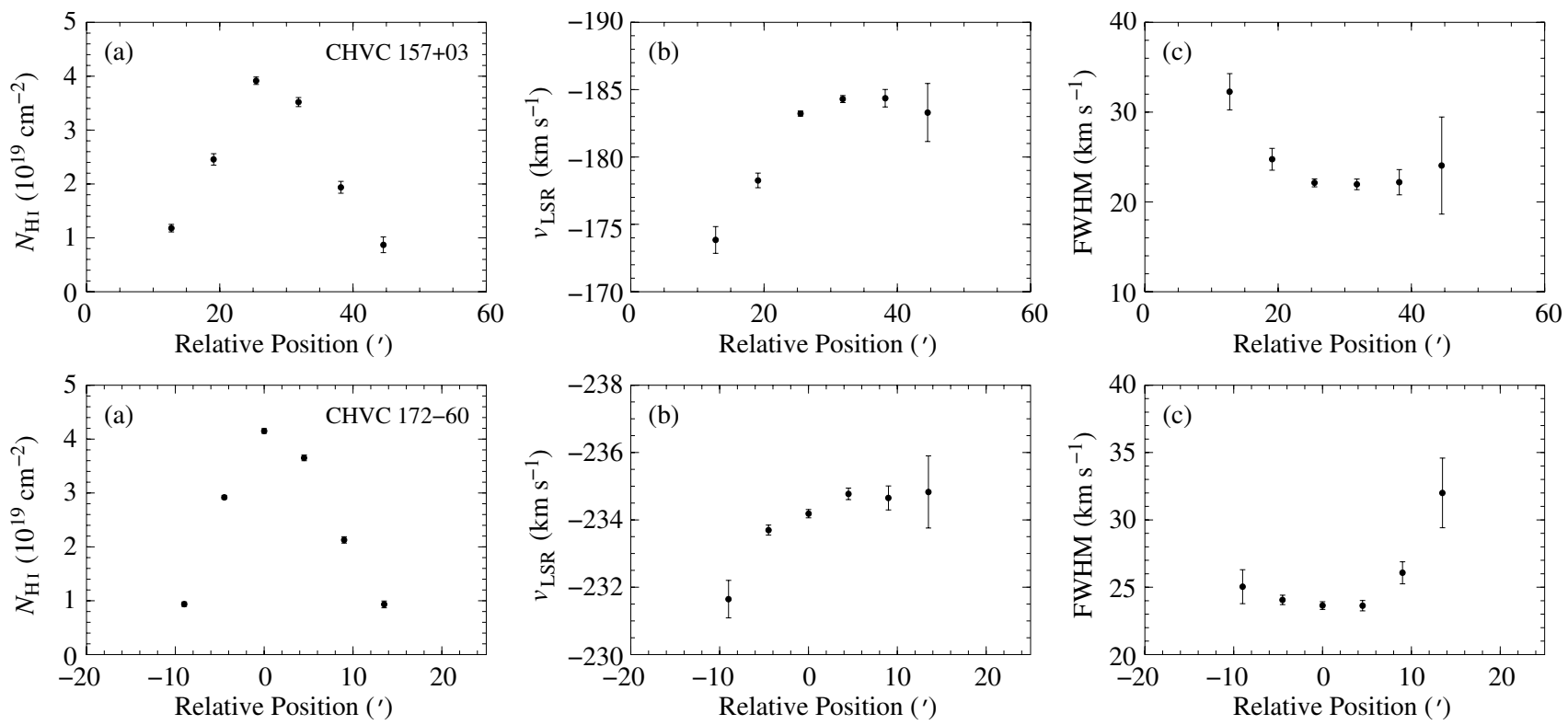

Fig. 7. Bow-shock shaped CHVCs as described in Sect. 4.4. a) Integrated H I column density along the slice indicated by the black crosses in Fig. 6a. b) Distribution of LSR radial velocities of the gas along the slice. c) Distribution of line widths (FWHM) along the slice.

deep spectra along the slice reveal a faint gas component which has even lower velocities of $v_{\mathrm{LSR}} \approx-110 \mathrm{~km} \mathrm{~s}^{-1}$. This results in a total velocity gradient of $85 \mathrm{~km} \mathrm{~s}^{-1}$ across the observed extent of the cloud. Furthermore, this velocity gradient resembles the flat rotation curve of a galaxy. This is already obvious in the velocity map in Fig. $8 \mathrm{~b}$ and is clearly illustrated by the velocity profile along the deep slice which was chosen along the velocity gradient and can be seen in Fig. 9b. This velocity gradient is accompanied by multiple line components especially in the central parts of CHVC $358+12$. The occurrence of such multiple components is traced by larger line widths of up to $60 \mathrm{~km} \mathrm{~s}^{-1} F W H M$ near the centre of the cloud. The properties of this remarkable $\mathrm{CHVC}$ will be discussed in more detail in a subsequent paper.

\section{Discussion}

\subsection{Evidence of ram-pressure interaction}

The most outstanding result of our observations is the morphological complexity of the investigated CHVCs. Ten out of eleven clouds reveal an obviously non-spherical morphology with four CHVCs appearing irregular. When taking spectra along an appropriate axis across each cloud, we originally intended to obtain sensitive and detailed column density profiles at the clouds' edges and to compare our observations with the results of the hydrodynamical simulations by Sternberg et al. (2002). But the explicitly irregular morphologies of most of the 11 observed CHVCs make a comparison of column density profiles and scale lengths nearly impossible. The simulations by Sternberg et al. (2002) were based on the assumption of spherically-symmetric and undisturbed gas clouds. In many cases, however, we see an obvious asymmetry in the Hi distribution which may be an indication of interaction effects between the CHVCs and an ambient medium.
The complex morphologies of the CHVCs in our sample are not a selection effect caused by the selection criteria discussed in Sect. 2. Virtually all of the 41 CHVCs in our previous, less sensitive Effelsberg survey (Westmeier 2003) disclose a non-spherical morphology, although the data were not sensitive enough to trace the distribution of the faint outer parts of the WNM. Thus, a complex, non-spherical distribution of H I column densities seems to be typical for the known population of CHVCs, and spherically-symmetric clouds like CHVC 148-82 are a rare exception.

The effects of ram-pressure stripping of HVCs were investigated by Quilis \& Moore (2001) using three-dimensional hydrodynamical simulations. They present pure gas clouds as well as dark-matter confined clouds in an ambient medium with a variety of different gas densities. They find that gas tails with H I column densities $\gtrsim 10^{19} \mathrm{~cm}^{-2}$ appear when the density of the ambient medium exceeds about $10^{-4} \mathrm{~cm}^{-3}$. Even with lower gas densities around $10^{-5} \mathrm{~cm}^{-3}$ faint gas tails emerge in their simulations with $\mathrm{H}$ I column densities of about $10^{18} \mathrm{~cm}^{-2}$.

The most prominent candidates for interacting clouds in our sample are the four head-tail CHVCs (see Figs. 4 and 5). The head-tail morphology of these objects already suggests the presence of ram pressure. This impression is additionally supported by the distribution of line widths. With the exception of CHVC 040+01, all head-tail CHVCs reveal a clear line width gradient with the narrowest $\mathrm{HI}$ lines appearing close to the head at the apparent leading edge of the cloud. This indicates a spatial separation of warm and cold neutral gas. In the case of CHVC 218+29, the bare cold gas lies exposed to its environment on one edge of the cloud with line widths around $4 \mathrm{~km} \mathrm{~s}^{-1}$ FWHM. A similar situation is observed for CHVC 017-25, where line widths of about $7 \mathrm{~km} \mathrm{~s}^{-1} F W H M$ are measured at the apparent leading edge.

Ram-pressure distortion is also a possible explanation for our two cases of bow-shock shaped CHVCs (see Figs. 6 and 7). 

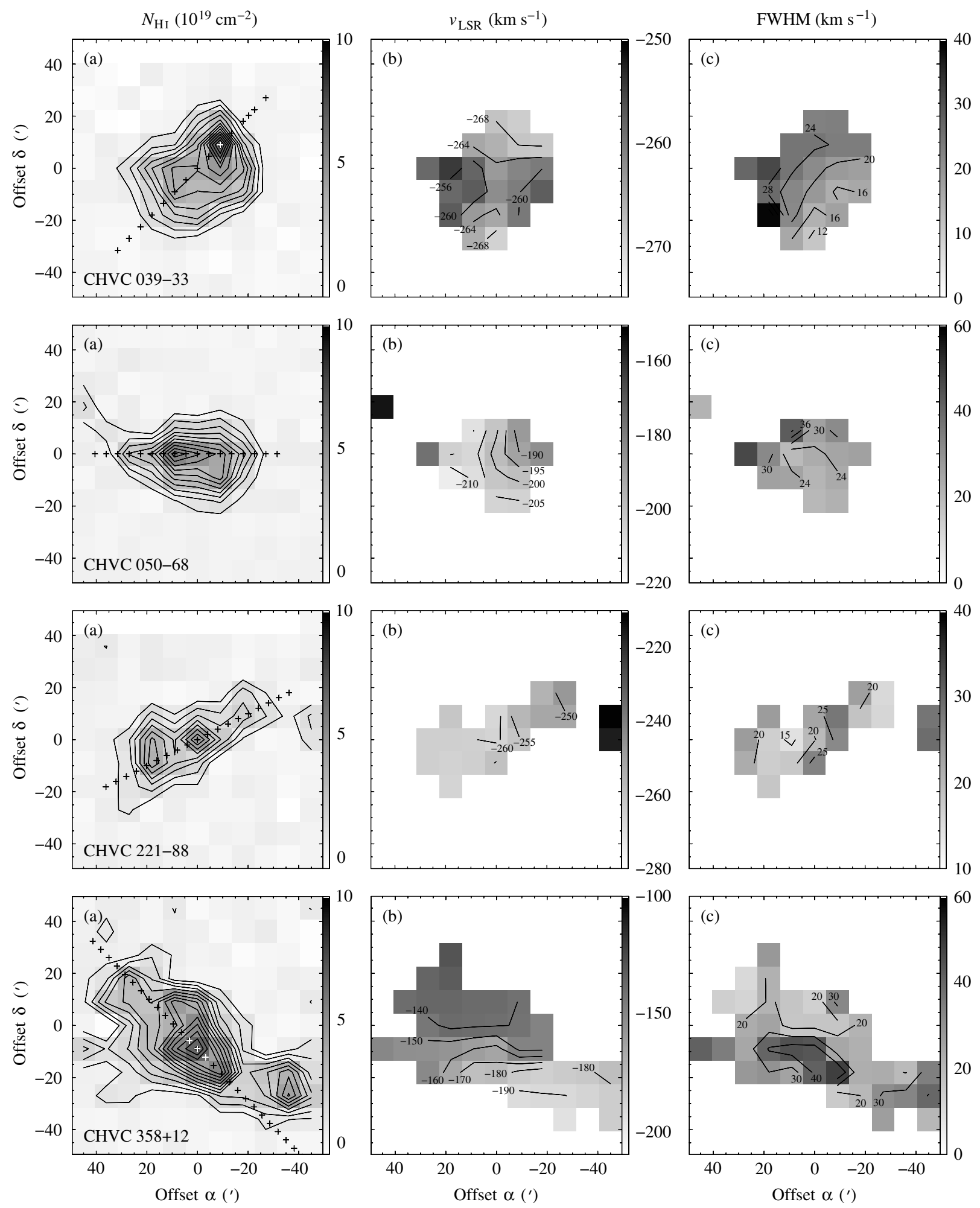

Fig. 8. Irregular CHVCs as described in Sect. 4.5. a) Integrated H I column density map. Contours start at $5 \times 10^{18} \mathrm{~cm}^{-2}$ with an increment of $5 \times 10^{18} \mathrm{~cm}^{-2}$. The crosses indicate the positions of individual spectra along the deep slice (see Fig. 9). b) Distribution of LSR radial velocities of the gas. c) Distribution of line widths (FWHM).

A detailed analysis of the distribution of radial velocities of the H I gas reveals a clear velocity gradient across both clouds. While radial velocities are more or less constant across most parts of the two CHVCs, they decrease significantly towards the presumed leading edges. This suggests a deceleration of the gas due to the ram pressure of the ambient medium. In the case of CHVC $157+03$, this velocity gradient measures about $10 \mathrm{~km} \mathrm{~s}^{-1}$ and is accompanied by a clear line width gradient with opposite sign. Line widths systematically increase towards the apparent leading edge which may indicate heating 

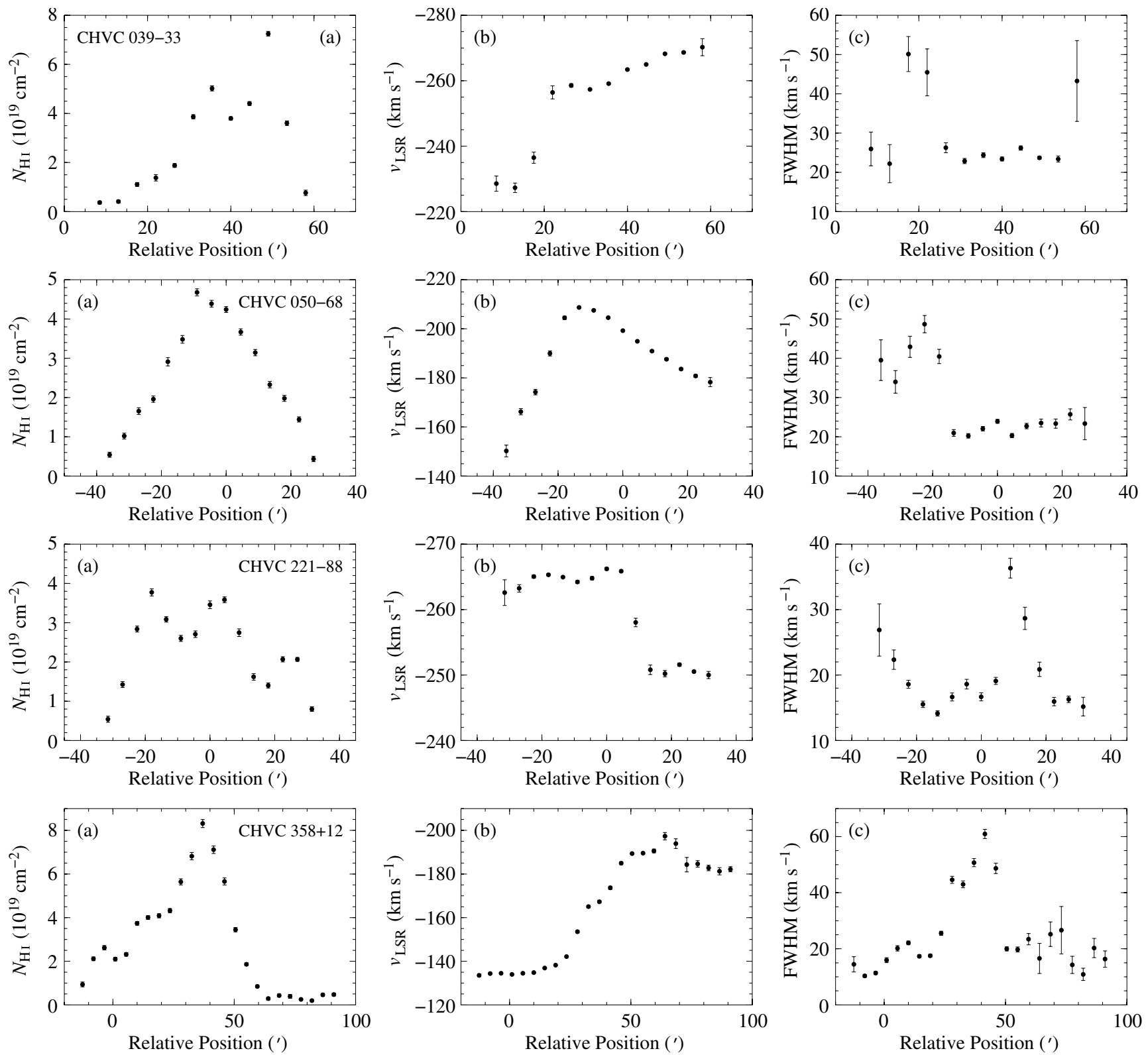

Fig. 9. Irregular CHVCs as described in Sect. 4.5. a) Integrated H I column density along the slice indicated by the crosses in Fig. 8a. b) Distribution of LSR radial velocities of the gas along the slice. c) Distribution of line widths $(F W H M)$ along the slice.

or growing turbulence of the gas in the course of ram-pressure interaction.

Burton et al. (2001) also reported significant asymmetries in the high column density regime $\left(N_{\mathrm{H}}>3 \times 10^{18} \mathrm{~cm}^{-2}\right)$ of their 10 CHVCs observed with the Arecibo telescope. In the low column density regime below about $3 \times 10^{18} \mathrm{~cm}^{-2}$, however, they found a high degree of reflection symmetry which they took as evidence against an external mechanism producing the observed asymmetries in the high column density cores. But their analysis is based only on one-dimensional drift scans at constant declination, whereas our deep slices were placed along the symmetry axis of each CHVC, making an interpretation in the sense of object symmetry more meaningful.

Another possible mechanism to produce the observed complex morphologies of most of our CHVCs is tidal interaction. Tidal forces, however, would act on both the compact cold cores and the diffuse warm envelopes in the same manner so that a misalignment between the core and the envelope, as in the case of head-tail CHVCs, cannot be explained by tidal interaction alone. But tidal forces could have shaped some of the irregular CHVCs in our sample. Clouds like CHVC 221-88 or CHVC 358+12 are highly elliptical, but they do not show any obvious misalignment between the diffuse envelope and the embedded cores, indicating that ram-pressure might not have been the principal process shaping these clouds.

Radiation pressure could also act on gas clouds and cause a misalignment between compact cores and diffuse envelopes. This process, however, can be excluded in the case of our CHVCs because it would require a strong radiation field as well as high density clumps with a significant amount of dust which has not yet been found in CHVCs. Wakker \& Boulanger (1986) investigated parts of the HVC complexes A and M in IRAS 

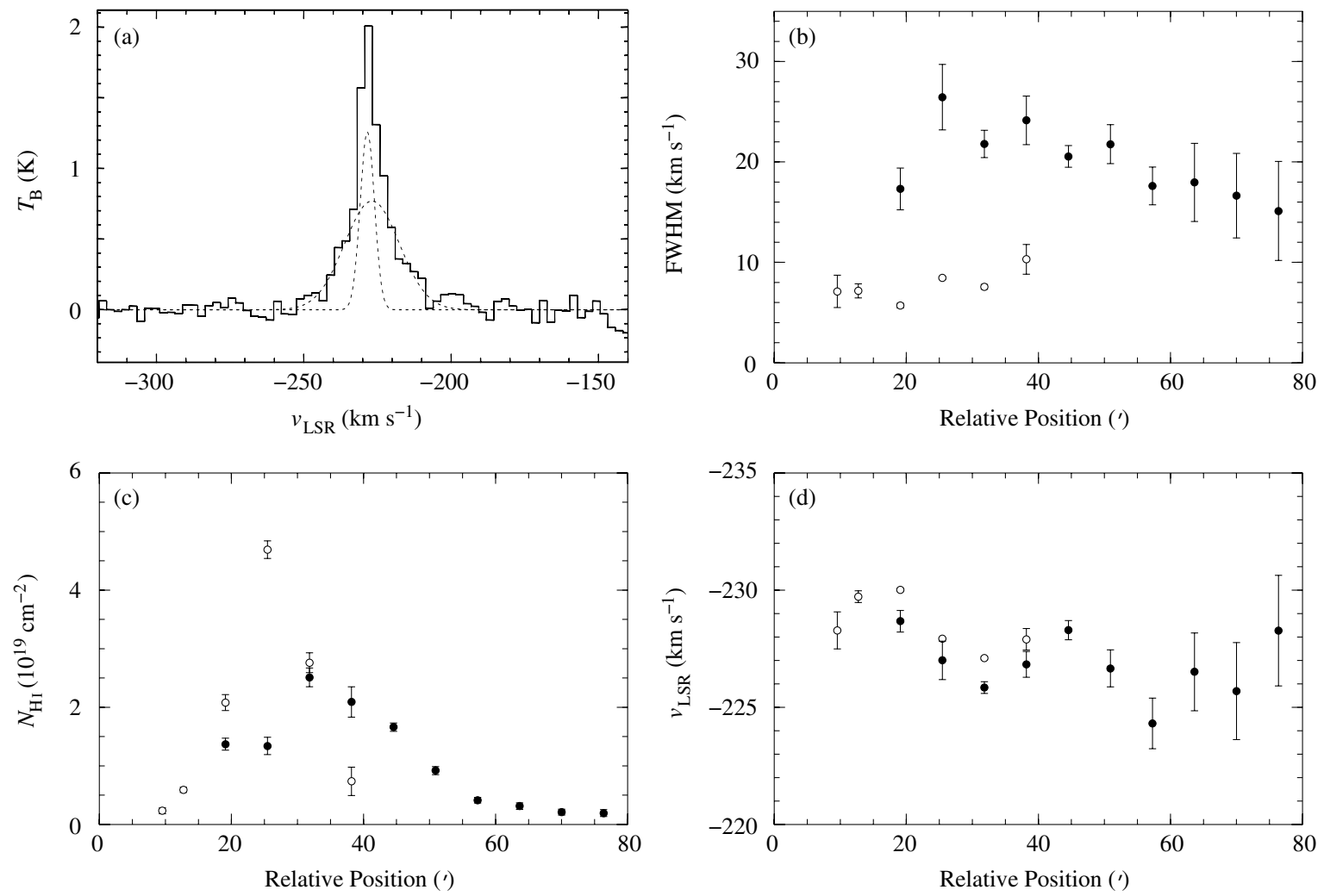

Fig. 10. CHVC 017-25. a) Typical spectrum near the centre of the cloud which shows a two-component line profile. The dotted curves represent the decomposition of the spectrum into two Gaussian components. The three diagrams show the distribution of b) line widths, c) H I column densities and d) LSR radial velocities along the deep slice for the cold (०) and warm (•) gas component. The error bars indicate the statistical uncertainties of the Gaussian fit.

observations, but they did not detect any dust in these HVCs, concluding that either the dust was too cold to be detected or that the dust abundance was below their detection limit. CHVCs have even lower H I peak column densities than the large HVC complexes so that the presence of large amounts of dust is not expected if one assumes the same dust properties as for the low-velocity H I clouds in the Galaxy. In addition, the strong radiation field should ionise the part of the CHVCs being exposed to the radiation. This should result in high $\mathrm{H} \alpha$ intensities which are not consistent with the faint $\mathrm{H} \alpha$ emission found by Tufte et al. (2002) and Putman et al. (2003).

\subsection{CHVC 017-25 as a prime example of ram-pressure interaction}

The most compelling example of a presumably interacting cloud, CHVC 017-25, is discussed in more detail at this point as the effects of ram-pressure distortion are quite pronounced and representative of other clouds in our sample. As already mentioned in Sect. 4.3.1, the head-tail structure of CHVC 017-25 does not seem very prominent at first glance. A more detailed analysis of the deep spectra along the apparent symmetry axis, however, provides evidence of a possible rampressure distortion of the entire cloud. Many spectra around the central part of CHVC 017-25 show a more complex H I line profile, indicating the superposition of two distinct gas components. Figure 10a presents an example spectrum in which a narrow line component $\left(\Delta v \approx 7 \mathrm{~km} \mathrm{~s}^{-1} F W H M\right)$ of cold neutral gas $(T \lesssim 1100 \mathrm{~K})$ seems to be superposed on a broad component $\left(\Delta v \approx 20 \mathrm{~km} \mathrm{~s}^{-1} F W H M\right)$ of warm neutral gas $(T \lesssim 9000 \mathrm{~K})$. Therefore, we decomposed the Hi line profiles along the deep slice across CHVC 017-25, where possible, into two Gaussian components.

Of course, such a Gaussian decomposition is an approximation as we will never meet two completely distinct gas phases with uniform temperatures. As de Heij et al. (2002a) have already pointed out, a decomposition of spectral lines into two Gaussian components, therefore, does not necessarily refer to two physically distinct systems. Nonetheless, the homogeneous physical parameters of the two line components justify the distinction of a cold and a warm gas phase as long as we only consider the qualitative properties of these two gas phases and their implications.

The results of the Gaussian decomposition of the spectral lines of CHVC 017-25 are presented in Figs. 10b-d. In Fig. 10b we have plotted the line widths of the two gas components as a function of relative position along the deep slice. The two gas phases are clearly separated by their different line widths of $\Delta v \approx 6 \ldots 10 \mathrm{~km} \mathrm{~s}^{-1} F W H M$ for the cold gas and $\Delta v \approx 16 \ldots 26 \mathrm{~km} \mathrm{~s}^{-1} F W H M$ in the case of the warm gas component. Figure 10c shows the column density profiles of both gas phases along the slice. The cold gas phase forms a compact head while the warm gas component is spatially much more extended so that we can speak of a compact, cold core 
embedded in a diffuse, warm envelope. The most remarkable result is that both gas phases are spatially separated from each other. At the south-eastern edge of the cloud only the narrow lines of the cold gas component can be seen. Towards the northwestern edge we can only trace the warm gas phase which forms an extended, faint tail and justifies our classification of CHVC 017-25 as a head-tail CHVC. This clear spatial separation of the cold core and the warm envelope could be explained if the envelope is being stripped off the compact core by the ram pressure of an ambient medium through which CHVC 017-25 is moving.

In Fig. 10d we have plotted the radial velocities of the two gas components across the deep slice. There is no clear gradient but a semi-periodic variation of radial velocities along the major axis of the cloud. At the four positions where both the cold and warm gas phases have been detected the velocity of the cold gas seems to be systematically higher (in absolute value) than that of the warm gas. This would be consistent with the idea of the cold core moving faster than the stripped-off warm envelope if we assume an infall of the cloud onto the Galaxy which is suggested by the highly negative radial velocity of $-171 \mathrm{~km} \mathrm{~s}^{-1}$ in the GSR frame. But the velocity difference of about $1 \mathrm{~km} \mathrm{~s}^{-1}$ between the core and the envelope is significantly smaller than the $2.6 \mathrm{~km} \mathrm{~s}^{-1}$ velocity resolution of our spectra so that this result is not reliable.

\subsection{Distance of CHVCs}

The most controversial parameter of CHVCs is their distance. Distance is the key to most of the physical parameters like the H I mass, the size, and, naturally, the overall distribution of the observed CHVC population within the Local Group. As the distance of CHVCs cannot be determined directly we have to apply indirect distance estimates. Burton et al. (2001) used an estimate of the thermal pressure at the interface of cold and warm neutral medium to obtain distances between 150 and $850 \mathrm{kpc}$ for a number of CHVCs observed with the Arecibo telescope. Such distances would place the CHVC population throughout the entire Local Group. Brüns et al. (2001) applied the virial theorem to a compact clump within CHVC $125+41$ to estimate a distance of the cloud of the order of $130 \mathrm{kpc}$. All these indirect distance determinations, however, require the assumptions of spherical symmetry and dynamical equilibrium of the cloud. We have seen that most of the 11 investigated CHVCs have a clearly non-spherical appearance and look highly disturbed so that in these cases simple distance estimates cannot be applied.

Evidence for a distribution of CHVCs within the Milky Way halo was found by Tufte et al. (2002). They detected $\mathrm{H} \alpha$ emission towards four out of five CHVCs investigated with the Wisconsin H-Alpha Mapper. The $\mathrm{H} \alpha$ intensities are comparable to those expected for clouds in the Galactic halo where the radiation field is strong enough to ionise the gas (see also Weiner et al. 2001). The metagalactic radiation field throughout the Local Group would be too weak to account for the detected $\mathrm{H} \alpha$ intensities. Putman et al. (2003) were able to confirm these results with the detection of $\mathrm{H} \alpha$ emission towards two other
CHVCs. These $\mathrm{H} \alpha$ measurements suggest that CHVCs might constitute a circumgalactic population with distances of only some $10 \mathrm{kpc}$ from the Galaxy.

Our only cloud with a spherically-symmetric appearance is CHVC 148-82. We can try to derive a rough distance estimate for this object by applying the following assumptions. Let us consider the cloud to be spherically-symmetric with radius $R$ and a constant mass density $\varrho=N_{\mathrm{H}} m_{\mathrm{H}} /(2 R f)$, where $N_{\mathrm{H}}$ is the central H I column density, $m_{\mathrm{H}}$ is the mass of a hydrogen atom, and the factor $f \equiv M_{\mathrm{HI}} / M$ has been introduced to account for additional mass components such as ionised gas or dark matter which are not traced by the H I column density. The total mass of the cloud is $M=(4 / 3) \pi R^{3} \varrho$. Furthermore, we consider CHVC 148-82 to be gravitationally bound and in dynamical equilibrium so that the virial theorem can be applied. Under the above assumptions the virial theorem reads

$M\left\langle v^{2}\right\rangle=\frac{3}{5} \frac{G M^{2}}{R}$

where $G$ is the gravitational constant, and $\left\langle v^{2}\right\rangle$ denotes the mean-square velocity of the particles which is related to the observed $F W H M \Delta v$ of the spectral lines by $\left\langle v^{2}\right\rangle=3 \Delta v^{2} /(8 \ln 2)$. We can now solve Eq. (1) for the distance $d$ of the cloud:

$d=\frac{15}{16 \pi \ln 2 G m_{\mathrm{H}}} \frac{f \Delta v^{2}}{N_{\mathrm{H}} \vartheta}$.

Here, $\vartheta$ denotes the angular radius of the cloud. In the case of CHVC 148-82, the average line width is $\Delta v \approx 20 \mathrm{~km} \mathrm{~s}^{-1}$ $F W H M$, the observed peak column density is $N_{\mathrm{HI}} \approx 8 \times$ $10^{19} \mathrm{~cm}^{-2}$, and the angular diameter amounts $2 \vartheta \approx 40^{\prime}$. Inserting these values into Eq. (2) results in a distance estimate for CHVC $148-82$ of $d \approx f \cdot 10 \mathrm{Mpc}$. Considering the expected additional amount of helium of $M_{\mathrm{He}} \approx 0.4 M_{\mathrm{H} \text { I }}$ (i.e. $f \approx 0.7$ ), CHVC 148-82 would lie at a distance of about $7 \mathrm{Mpc}$ and would have a total mass of $3.2 \times 10^{9} M_{\odot}$ and a radius of about $40 \mathrm{kpc}$. These parameters are highly implausible. A closer distance can be achieved by assuming a significant amount of additional mass (e.g. ionised gas or dark matter) or by rejecting the assumption of virialisation of CHVC 148-82. The problem that pure $\mathrm{HI}$ CHVCs cannot be gravitationally stable at reasonable distances was discussed in detail by Sternberg et al. (2002). They consider the possibility of an additional pressure support of CHVCs by the presence of a surrounding medium. The presence of such an ambient medium in which CHVCs are embedded is illustrated by the majority of disturbed clouds among our sample. We can account for an external pressure support by inserting an additional pressure term into the virial theorem which then reads

$M\left\langle v^{2}\right\rangle=\frac{3}{5} \frac{G M^{2}}{R}+3 P V$.

Here, $V=(4 / 3) \pi R^{3}$ is the volume of the cloud, and $P$ denotes the external pressure. This equation, however, is only valid for clouds without any dark matter that is not governed by an external pressure component. Solving Eq. (3) for $P$ yields

$P=\frac{m_{\mathrm{H}} N_{\mathrm{H}} \Delta v^{2}}{16 \ln 2 f \vartheta d}-\frac{\pi G m_{\mathrm{H}}^{2} N_{\mathrm{H} I}^{2}}{15 f^{2}}$ 


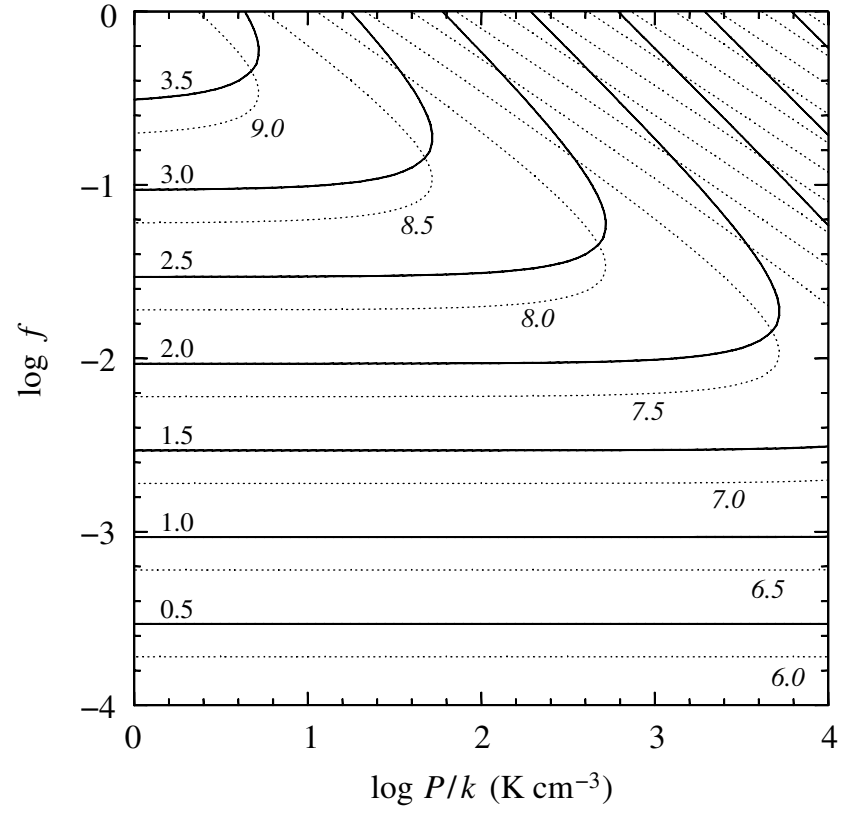

Fig. 11. Constraining the distance of CHVC 148-82. Distance $(\log d / \mathrm{kpc}$, solid contours, labelled from 0.5 to 3.5$)$ and equilibrium mass $\left(\log M / M_{\odot}\right.$, dotted contours, labelled from 6.0 to 9.0 but going down to 2.5 in the upper right corner of the diagram) plotted as a function of external pressure $P / k$ and $\mathrm{HI}$ mass fraction $f \equiv M_{\mathrm{H}} / M$ according to Eq. (4). In the most reasonable parameter range of $\log f \approx-2 \ldots 0$ and $\log P / k \approx 1 \ldots 3$ the distance of CHVC $148-82$ is not well constrained and has values between $30 \mathrm{kpc}$ and $2 \mathrm{Mpc}$.

According to Eq. (4), the distance of the cloud is influenced by two free parameters, the external pressure $P$ and the H I mass fraction $f$. In Fig. 11 we have plotted the mass $M$ (dotted contours) and the distance $d$ (solid contours) of CHVC 148-82 as a function of these two parameters according to Eq. (4). For very small $f \lesssim 10^{-2}$ both distance and mass of the cloud become independent of the external pressure because stabilisation of the cloud is by far dominated by the mass. In this case, the distance would be $d \lesssim 100 \mathrm{kpc}$ and the mass $M \lesssim 5 \times 10^{7} M_{\odot}$. For more realistic values of $f$ distance and mass strongly depend on the strength of the external pressure. In the most reasonable parameter range of $\log f \approx-2 \ldots 0$ and $\log P / k \approx 1 \ldots 3$ the distance of CHVC $148-82$ is not well constrained and has values between $30 \mathrm{kpc}$ and $2 \mathrm{Mpc}$. These results show that we cannot obtain any reliable distance estimate for CHVC 148-82 due to the major uncertainties in parameter space in combination with a number of doubtful assumptions such as constant mass density or virialisation of the cloud.

In other words, within a realistic range of values for the external pressure and the H I mass fraction we can derive any distance within the Local Group for CHVC 148-82. This makes earlier distance estimates, placing CHVCs throughout the entire Local Group, doubtful as they are governed by similar assumptions and uncertainties. On the other hand, we observe interaction effects in our data. Head-tail structures and bowshock shapes are suggestive of ram-pressure interaction between CHVCs and the surrounding medium which may indicate a distribution of CHVCs in the vicinity of the Milky Way. Sternberg et al. (2002) simulated a circumgalactic population of CHVCs with distances of $150 \mathrm{kpc}$ from the Milky Way. If we consider the same distance for CHVC 148-82 we would need an external pressure of $P / k \approx 180 \mathrm{~K} \mathrm{~cm}^{-3}$ according to Eq. (4) to stabilise the cloud in the case of no additional dark matter and gas content except helium. This seems to be a rather high external pressure for such large distances from the Galaxy. Any dark matter content, however, will additionally stabilise CHVC 148-82 and decrease the required amount of external pressure.

Furthermore, there are indications for the existence of a hot ionised intergalactic medium which could provide an additional pressure stabilisation of CHVCs. Rasmussen et al. (2003) used X-ray absorption lines of O VII, O VIII, C VI, and Ne IX detected against three active galactic nuclei to constrain the physical properties of the absorbing gas. They obtained an upper limit for the electron density of the ionised medium of $n_{\mathrm{e}}<2 \times 10^{-4} \mathrm{~cm}^{-3}$, corresponding to a thermal pressure of $P / k \lesssim 770 \mathrm{~K} \mathrm{~cm}^{-3}$ with a gas temperature of $2 \times 10^{6} \mathrm{~K}$. Following limitations in the possible scale lengths of the absorber, Rasmussen et al. (2003) were able to locate the absorbing gas within the Local Group with a lower limit for the scale length of about $140 \mathrm{kpc}$. Even if the pressure provided by this ionised intergalactic medium is not sufficient to support CHVCs alone, a combination of external pressure and dark matter content could stabilise clouds like CHVC 148-82 even at distances of the order of $100 \mathrm{kpc}$ from the Galaxy.

The above results and considerations lead to a consistent picture in which CHVCs might constitute a circumgalactic population with typical distances of the order of $100 \mathrm{kpc}$ from the Milky Way. At such distances, CHVCs could be embedded in a hot ionised intergalactic gas or an extended Galactic corona and be distorted by ram-pressure interaction with this ambient medium. Furthermore, the external pressure provided by the ambient medium could support CHVCs in addition to their own gravitational potential.

\section{Summary}

We have mapped 11 compact high-velocity clouds (CHVCs) in the $21-\mathrm{cm}$ line emission of neutral, atomic hydrogen, using the Effelsberg 100-m radio telescope. We obtained a baseline rms of roughly $\sigma_{\text {rms }}=50 \mathrm{mK}$ at the original velocity resolution of $2.6 \mathrm{~km} \mathrm{~s}^{-1}$, leading to a 1-sigma H I column density detection limit of about $2.4 \times 10^{17} \mathrm{~cm}^{-2}$ per spectral channel. These maps allow us to examine the overall morphology of the clouds. In addition, we have obtained deep spectra along the symmetry axis across each $\mathrm{CHVC}$ with denser angular sampling. Along these deep slices we reach a baseline rms of $\sigma_{\mathrm{rms}} \approx 25 \ldots 35 \mathrm{mK}$ at the original velocity resolution of $2.6 \mathrm{~km} \mathrm{~s}^{-1}$, resulting in an average 1-sigma H I column density detection limit of about $1.2 \ldots 1.7 \times 10^{17} \mathrm{~cm}^{-2}$ per spectral channel. These deep slices allow us to extract the column density profile in great detail as well as the velocity and line width gradient across each cloud.

The most outstanding result of our observations is the complexity of the investigated CHVCs. The numerous head-tail structures and bow-shock shapes point out that the head-tail structure of CHVC 125+41 found by Brüns et al. (2001) is not 
unique. Among our 11 objects we found only one cloud with a spherically-symmetric appearance. Four CHVCs have a headtail structure, two CHVCs show a bow-shock shape, and the remaining four clouds are irregular. The complex morphologies of most of our 11 CHVCs suggest that they may be disturbed by the ram pressure of the surrounding medium. In cases like CHVC 017-25, the diffuse envelope of warm neutral gas appears to be stripped off the compact cold core of the cloud, forming a more or less extended, faint $\mathrm{HI}$ tail with column densities of typically $N_{\mathrm{HI}} \lesssim 10^{19} \mathrm{~cm}^{-2}$. Some of the irregular CHVCs in our sample are highly elliptical but do not show a noticeable misalignment between the diffuse envelope and the embedded clumps. In these cases, tidal interactions instead of ram pressure might have played a significant role in shaping the clouds.

The existence of an ambient medium around CHVCs can also solve the problem of stability. If CHVCs were only made of $\mathrm{HI}$ they would not be gravitationally stable at distances of less than a few Mpc. At such large distances, however, CHVCs would be extremely large and diffuse objects with H I masses of a few times $10^{9} M_{\odot}$. Additional mass components like ionised gas, molecular gas or dark matter could solve this distance problem and help to stabilise CHVCs. Another option, however, is stabilisation by external pressure. We showed that at a distance of $150 \mathrm{kpc}$ an external pressure of $P / k \approx 180 \mathrm{~K} \mathrm{~cm}^{-3}$ would be sufficient to stabilise objects like CHVC 148-82 even without any additional mass components except for the expected associated helium content.

The above considerations show that our observations are consistent with a circumgalactic population of CHVCs with distances of the order of $100 \mathrm{kpc}$. At such distances, CHVCs can be embedded in the gaseous environment of an extended Galactic corona or an ionised intergalactic medium which not only stabilises the clouds but which can also provide the necessary ram pressure to account for the observed complex morphologies of most of our CHVCs.

Acknowledgements. We wish to thank our anonymous referee for many useful comments which helped to improve this paper. T.W. acknowledges support by the Deutsche Forschungsgemeinschaft (German Research Foundation) through project number KE757/4-1. The results presented in this paper are based on observations with the 100-m telescope of the MPIfR (Max-Planck-Institut für Radioastronomie) at Effelsberg.

\section{References}

Banks, T., Dodd, R. J., \& Sullivan, D. J. 1995, MNRAS, 272, 821

Barnes, D. G., Staveley-Smith, L., de Blok, W. J. G., et al. 2001, MNRAS, 322, 468

Blitz, L., Spergel, D. N., Teuben, P. J., Hartman, D., \& Burton, W. B. 1999, ApJ, 514, 818

Braun, R., \& Burton, W. B. 1999, A\&A, 341, 437

Braun, R., \& Burton, W. B. 2000, A\&A, 354, 853

Brüns, C., Kerp, J., Kalberla, P. M. W., \& Mebold, U. 2000, A\&A, 357,120

Brüns, C., Kerp, J., \& Pagels, A. 2001, A\&A, 370, L26

Brüns, C., Kerp, J., Staveley-Smith, L., et al. 2005, A\&A, 432, 45

Burton, W. B., Braun, R., \& Chengalur, J. N. 2001, A\&A, 369, 616

Hartmann, D., \& Burton, W. B. 1997, Atlas of Galactic Neutral Hydrogen (Cambridge University Press)

de Heij, V., Braun, R., \& Burton, W. B. 2002a, A\&A, 391, 67

de Heij, V., Braun, R., \& Burton, W. B. 2002b, A\&A, 391, 159

de Heij, V., Braun, R., \& Burton, W. B. 2002c, A\&A, 392, 417

Kalberla, P. M. W., Mebold, U., \& Reich, W. 1980, A\&A, 82, 275

Kalberla, P. M. W., Mebold, U., \& Reif, K. 1982, A\&A, 106, 190

Klypin, A., Kravtsov, A. V., Valenzuela, O., \& Prada, F. 1999, ApJ, 522,82

Moore, B., Ghigna, S., Governato, G., Lake, G., Quinn, T., et al. 1999, ApJ, 524, L19

Muller, C. A., Oort, J. H., \& Raimond, E. 1963, C. R. Acad. Sci. Paris, 257,1661

Pisano, D. J., Barnes, D. G., Gibson, B. K., et al. 2004, ApJ, 610, L17

Putman, M. E., de Heij, V., Staveley-Smith, L., et al. 2002, AJ, 123, 873

Putman, M. E., Bland-Hawthorn, J., Veilleux, S., et al. 2003, ApJ, 597, 948

Quilis, V., \& Moore, B. 2001, ApJ, 555, L95

Rasmussen, A., Kahn, S. M., \& Paerels, F. 2003, in The IGM/Galaxy Connection: The Distribution of Baryons at $z=0$, ed. J. L. Rosenberg, \& M. E. Putman, ASSL Conf. Proc., 281, 109

Sternberg, A., McKee, C. F., \& Wolfire, M. G. 2002, ApJS, 143, 419

Tufte, S. L., Wilson, J. D., Madsen, G. J., Haffner, L. M., \& Reynolds, R. J. 2002, ApJ, 572, L153

Wakker, B. P. 1991, A\&A, 250, 499

Wakker, B. P., \& Boulanger, F. 1986, A\&A, 170, 84

Wakker, B. P., \& van Woerden, H. 1997, ARA\&A, 35, 217

Weiner, B. J., Vogel, S. N., \& Williams, T. B. 2001, in Gas and Galaxy Evolution, ed. J. E. Hibbard, M. Rupen, \& J. H. van Gorkom, ASP Conf. Proc., 240, 515

Westmeier, T. 2003, Diploma thesis, Rheinische Friedrich-WilhelmsUniversität Bonn

Zwaan, M. 2000, Ph.D. Thesis, Rijksuniversiteit Groningen 